\title{
DRAINED ASH SWAMP \\ (VERATRO ALBI-FRAXINETUM ANGUSTIFOLIAE), A NEW ASSOCIATION IN THE NYÍRSÉG, NE HUNGARY
}

\author{
B. KeVEY ${ }^{1}$, L. PAPP ${ }^{2}$ and G. Lendvai ${ }^{3}$ \\ ${ }^{1}$ Department of Ecology, University of Pécs, H-7624 Pécs, Ifjúság u. 6, Hungary \\ E-mail: keveyb@gamma.ttk.pte.hu \\ ${ }^{2}$ Botanical Garden, University of Debrecen, H-4032 Debrecen, Egyetem tér 1, Hungary \\ E-mail:papp.laszlo@gf.unideb.hu \\ ${ }^{3}$ H-7000 Sárbogárd, Tompa Mihály u. 38/C, Hungary; E-mail: gaborlendvai@hotmail.com
}

(Received 29 March, 2017; Accepted 8 August, 2018)

This paper presents the phytosociological description of a drained swamp community, Veratro albi-Fraxinetum angustifoliae, so far found only in the Nyírség at Nyírábrány "Kiskőrises", "Mogyorósi-erdo""; Vámospércs "Jónásrész-Kőrises"; and Vámospércs "Jónásrész-Buzita". The habitat of the community is transitional between that of alder swamps (Fraxino pannonicae-Alnetum glutinosae), and hardwood riparian forests (Fraxino pannonicae-Ulmetum). The community is characterised by high proportions of character species of Alnion glutinosae and Molinion coerulei as well as Quercetea pubescentis-petraeae s. 1., whereas character species of the order Fagetalia are almost completely absent. It hosts several rare, often threatened species, such as Angelica palustris, Ophioglossum vulgatum, Trollius europaeus and Veratrum album.

Key words: ash swamp, Great Hungarian Plain, Natura 2000, nature reserve, syntaxonomy

\section{INTRODUCTION}

Hardwood riparian forests have been the subject of our long-term research on the distribution, composition and geographical variation of the forest vegetation in Hungary. Hardwood riparian forests still occur in most lowland areas of Hungary under different environmental conditions and thus are particularly suitable for studying phytosociological differentiation and biogeographical relationships in the forest vegetation within the Carpathian Basin. During our work in the Nyírség, an extensive area in the northeastern part of Hungary covered with eolic sand, we found several Fraxinus angustifolia dominated forest stands that seemed to be different from all known forest communities described previously in the country (Figs 1-2). No similar forest community is known to exist in the European vegetation either (BraunBlanquet 1964, Ellenberg 1986, Horvat 1938, Horvat et al. 1974, Mucina et al. 1993, Oberdorfer 1992a, b, Rodwell et al. 2002, Willner and Grabherr 2007a, b).

Because their species composition appeared to be substantially different from that of the Fraxino pannonicae-Ulmetum Soó in Aszód 1935 corr. Soó 
1963, a community once widely distributed in similarly moist habitats across the country, and Fraxino pannonicae-Alnetum Soó et Járai-Komlódi in JáraiKomlódi 1958, we described it as a novel association under the name Veratro albi-Fraxinetum angustifoliae Kevey et Papp L. (Kevey 2008).

Unfortunately, this association has not been described in more detail, nor has a synoptic table of it been published since then. Here we are going to fill this gap by providing a detailed description of the community based on our sample material of ten relevés. Our primary goal is to substantiate the split of this new association from the rest of the hardwood riparian forests found in Hungary.

\section{MATERIAL AND METHODS}

\section{Research area}

The studied Fraxinus angustifolia dominated stands were found in four different forest areas in the Nyírség: the Mogyorósi-erdő and Kis-kőrises near the town of Nyírábrány, and the Jónásrész-Kőrises and Jónásrész-Buzita near the village of Nyíracsád. The stands were all found along the edge of local

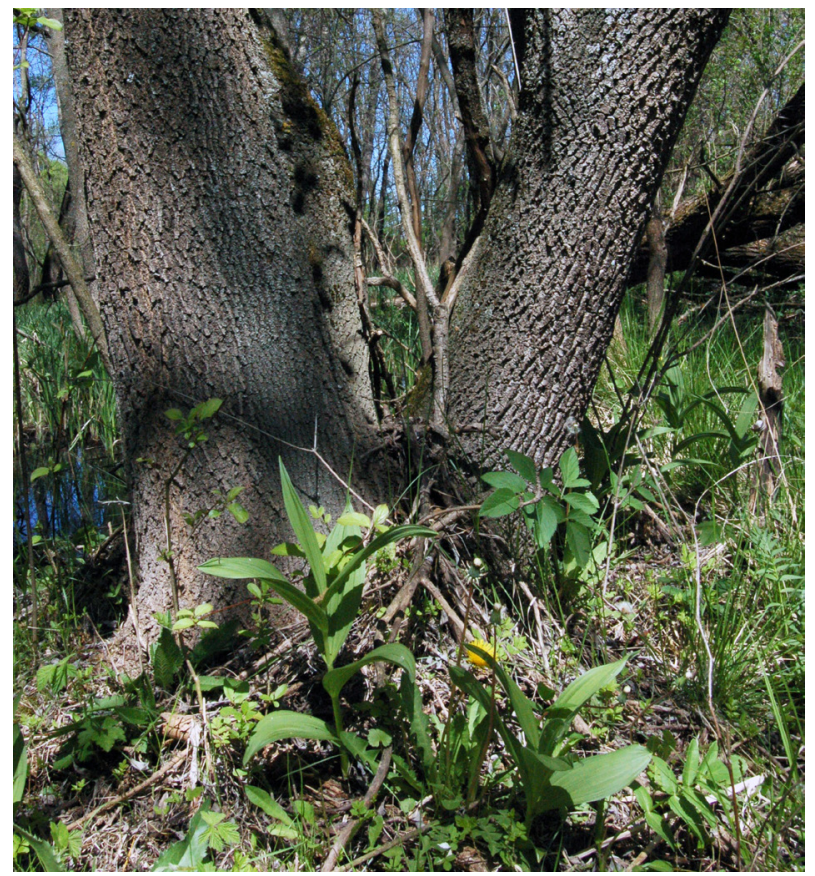

Fig. 1. Veratro albi-Fraxinetum angustifoliae: Vámospércs “Jónásrész" with Veratrum album in the foreground (photo: L. Papp) 
depressions where the habitat is characterised by high groundwater levels, which rises above the ground surface only in very wet periods. They either grow at the fringe of genuine alder swamps (Fraxino pannonicae-Alnetum) in the deepest parts of local depressions, or form the transition zone between these swamps and oak-ash-elm forests (Fraxino pannonicae-Ulmetum) growing on higher ground (Fig. 3). Since the ground is normally not covered with water throughout most of the vegetative period in their habitats, the soil contains only small amounts of peat that is generally decaying.

The three studied forest areas are all parts of the Natura 2000 network, and are also protected by national law. The Jónásrész "Kőrises" and "Buzita", and "Kis-kőrises" are state reserves, whereas the "Mogyorósi-erdo"" is under strict protection.

\section{Methods}

Our sampling procedure followed the traditional quadrate method of the Zürich-Montpellier phytosociological school (Becking 1957, Braun-Blanquet 1964). Sample plots were designated visually by selecting the parts of a stand that seemed to be the most homogeneous in habitat characteristics,

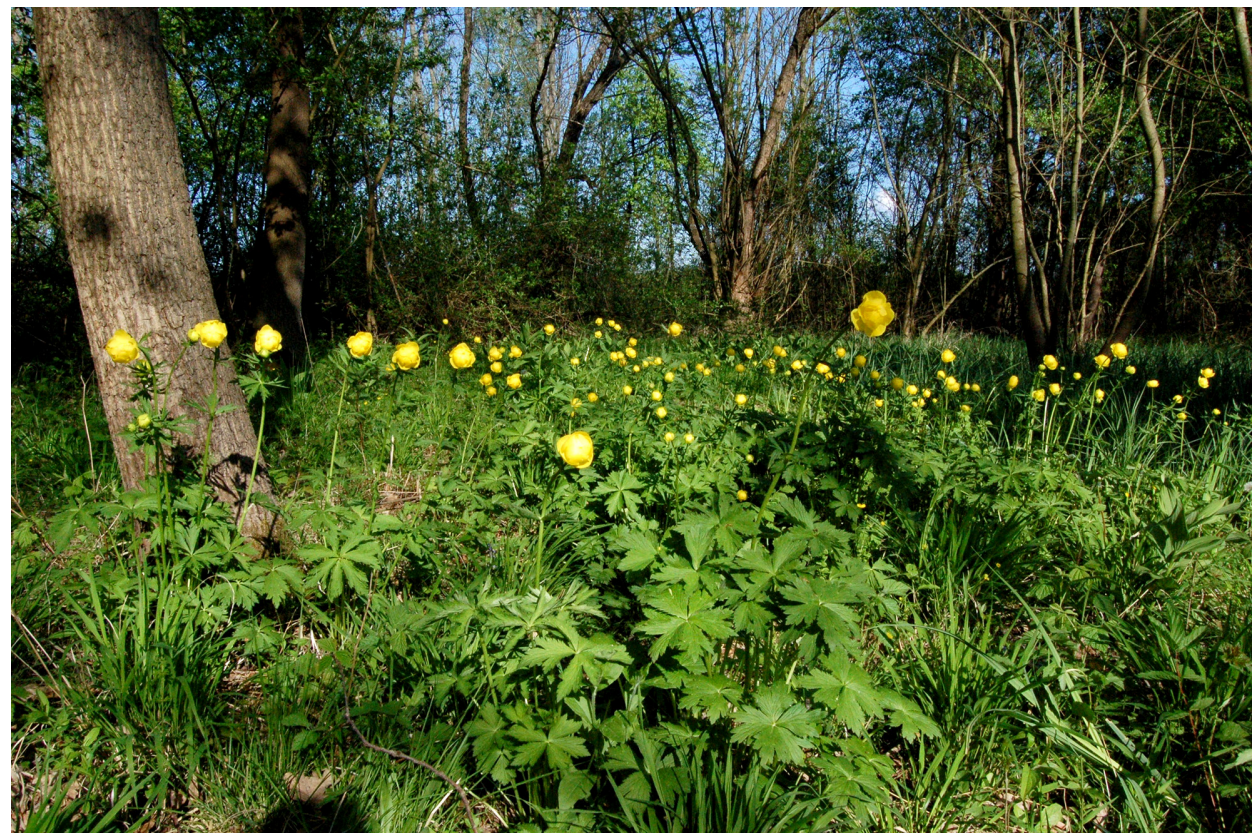

Fig. 2. Veratro albi-Fraxinetum angustifoliae: Vámospércs "Jónásrész" with Trollius europaeus in the herb layer (photo: L. Papp) 
vegetation structure and species composition, and showed no signs of human impact (including forest management). Because the traditionally used $400 \mathrm{~m}^{2}$ plot size does not satisfy the requirement for minimal area (see Du Rietz 1921), our sample plots were $1600 \mathrm{~m}^{2}$ in size except for one $\left(1200 \mathrm{~m}^{2}\right)$. This size suffices the requirement of representativity in temperate deciduous forests (Kevey 2008).

Because forests in similarly mesic habitats tend to exhibit large phenological changes during the vegetative period, we sampled each stand twice (spring and summer) using the same plots. During sampling, we recorded all species within the sample quadrate and estimated their projected cover. We also estimated the height of each vegetation layer, and the trunk diameter of trees.

The raw data were compiled and arranged in a synoptic table by the NS software program (Kevey and Hirmann 2002), which also was used to calculate constancy values of each species, and proportions of species characteristic of a particular syntaxon. To assess the syntaxonomic relationship of the studied stands, we compared them to a representative material of the spatially adjacent Fraxino pannonicae-Alnetum and Fraxino pannonicae-Ulmetum, and all previously described communities growing in similar habitats in Hungary: Ophioglosso-Betuletum pubescentis (Vértesalja: Riezing and Szollát 2008-2009: 6 relevés); Molinio-Alnetum glutinosae (Tengelici-homokvidék: Kevey 2008: 20 relevés); Molinio-Salicetum cinereae (Szigetköz: Kevey 2008: 25 relevés). In doing so, we performed pairwise comparisons between sample sets and deter-

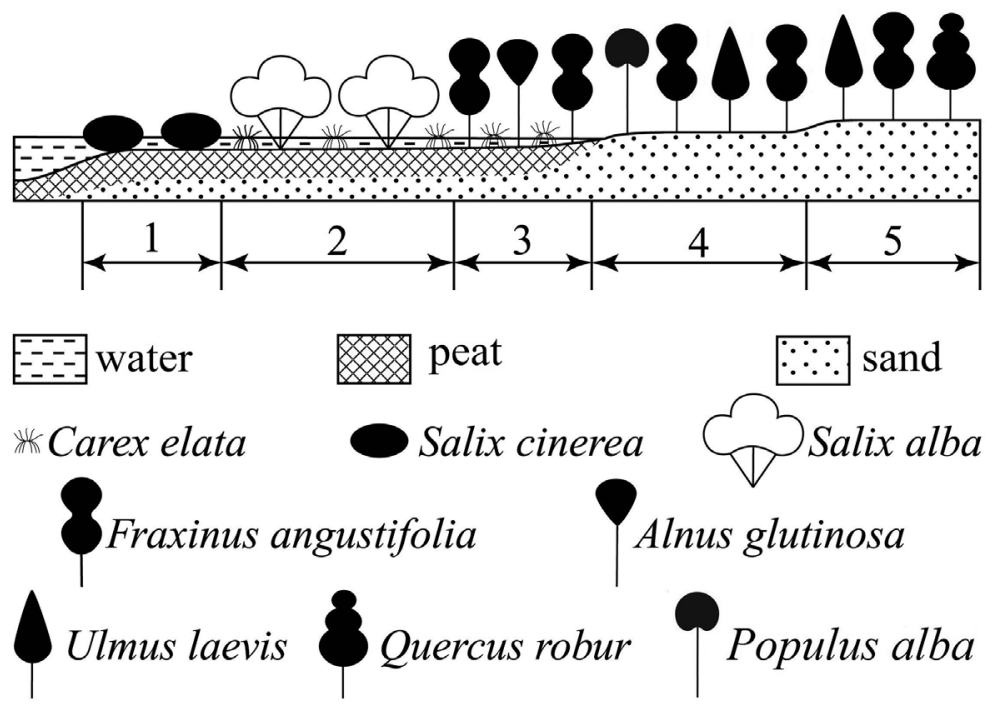

Fig. 3. Vegetation diagram of the "Mogyorósi-erdo"" at Nyírábrány. 1 = Calamagrostio-Salicetum cinereae; 2 = Carici elatae-Salicetum albae; 3 = Fraxino pannonicae-Alnetum glutinosae; $4=$

Veratro albi-Fraxinetum angustifoliae; 5: Fraxino pannonicae-Ulmetum. (Kevey original) 
mined the set of differential species (species that differed in their constancy value by at least two steps) and the proportions of character species. We also carried out binary cluster and principal coordinates analyses (PCoA) with the help of the Syntax 2000 package (Podani 2001). The method of grouping in the cluster analyses was complete link, and the similarity coefficient in both types of analyses was that of Baroni-Urbani and Buser.

The names of plants and syntaxonomic categories follow the nomenclature of Király (2009) and that of Borhidi and Kevey (1996), Borhidi et al. (2012), and Kevey (2008), respectively. Designation of species as character species of phytosociological taxa is primarily adopted from Soó $(1964,1966,1968,1970,1973$, 1980 ) with some modifications based on more recent literature (see Borhidi 1993, 1995, Horváth et al. 1995) and our own research experience (Kevey ined.).

The order of syntaxa in the synoptic and statistical tables follows the modified syntaxonomic system of Soó (1980) according to the suggestions and results of Borhidi et al. (2012), Kevey (2008), Mucina et al. (1993) and Oberdorfer $(1992 a, b)$.

\section{RESULTS}

\section{Physiognomy and structure}

In the studied stands, the forest canopy was structured into two distinct layers. The upper layer was situated at about 20-28 $\mathrm{m}$ height and was rather dense with high (60-80\%) projected cover. The most abundant (A-D: $3-4)$ tree species in this layer were Fraxinus angustifolia subsp. danubialis and Populus alba. They were also constant species across the samples.

The lower canopy layer was at the height of $12-20 \mathrm{~m}$. The projected cover was rather variable among samples. It was made up of mostly tree-sized shrubs and young individuals of trees. The most abundant species in this layer was Fraxinus angustifolia subsp. danubialis.

Shrubs in the samples were 1.5-3.5 $\mathrm{m}$ tall, and formed a moderately dense layer with $25-70 \%$ cover value. It was composed of Cornus sanguinea, Crataegus monogyna, Frangula alnus, Fraxinus angustifolia subsp. danubialis, and Ligustrum vulgare. Only Cornus sanguinea and Ligustrum vulgare had rather high cover values. The layer of saplings was greatly variable in projected cover $(1-50 \%)$.

All samples had an apparent herbaceous layer, which greatly varied in cover (35-85\%). The most frequent species include Angelica sylvestris, Brachypodium sylvaticum, Cucubalus baccifer, Filipendula ulmaria, Geranium palustre, Listera ovata, Lychnis flos-cuculi, Lysimachia nummularia, Moehringia trinervia, Poa trivialis, Scrophularia nodosa, Valeriana officinalis, Veratrum album. Only two species (Brachypodium sylvaticum and Convallaria majalis) were locally abundant at places (Table 1). 


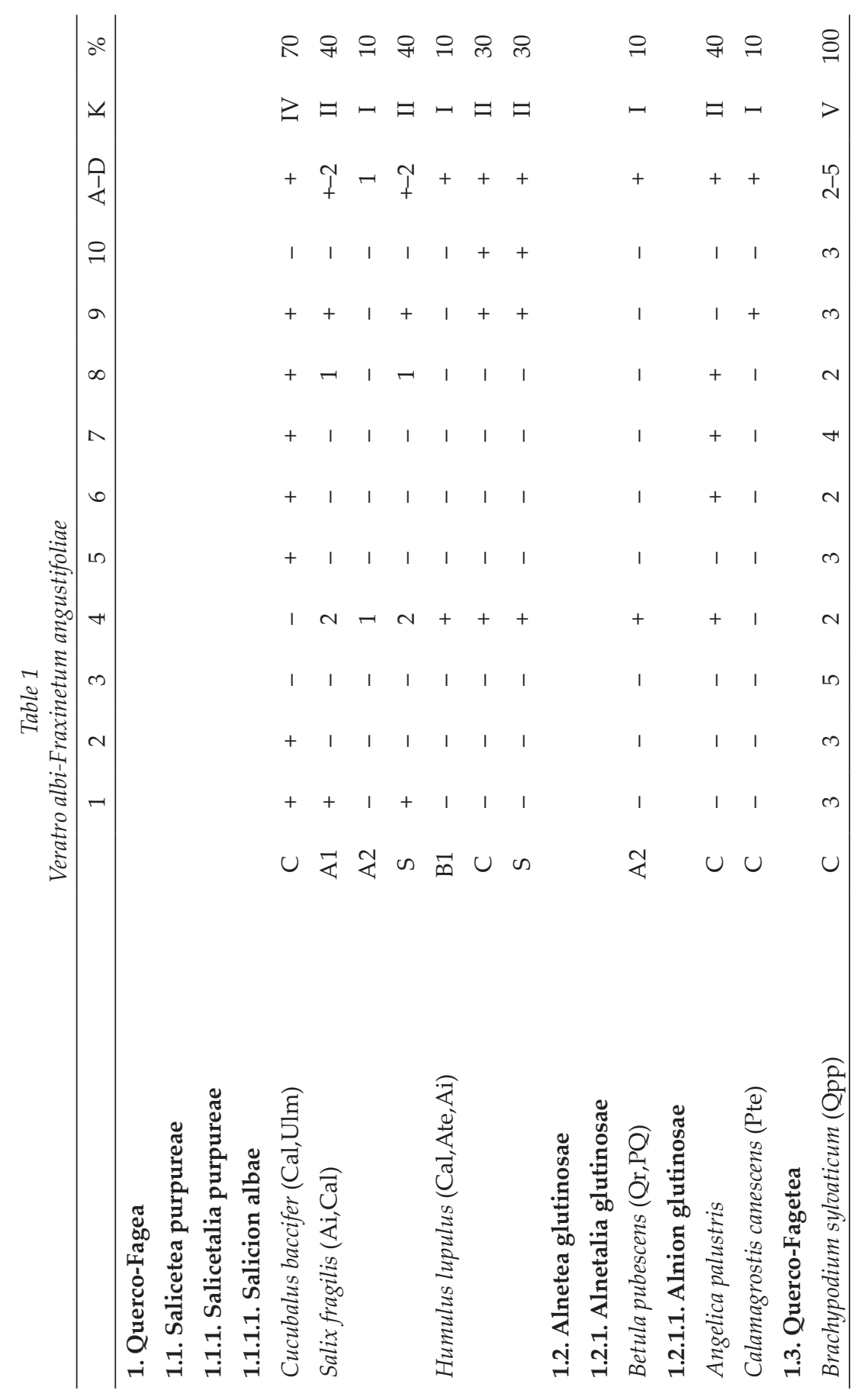




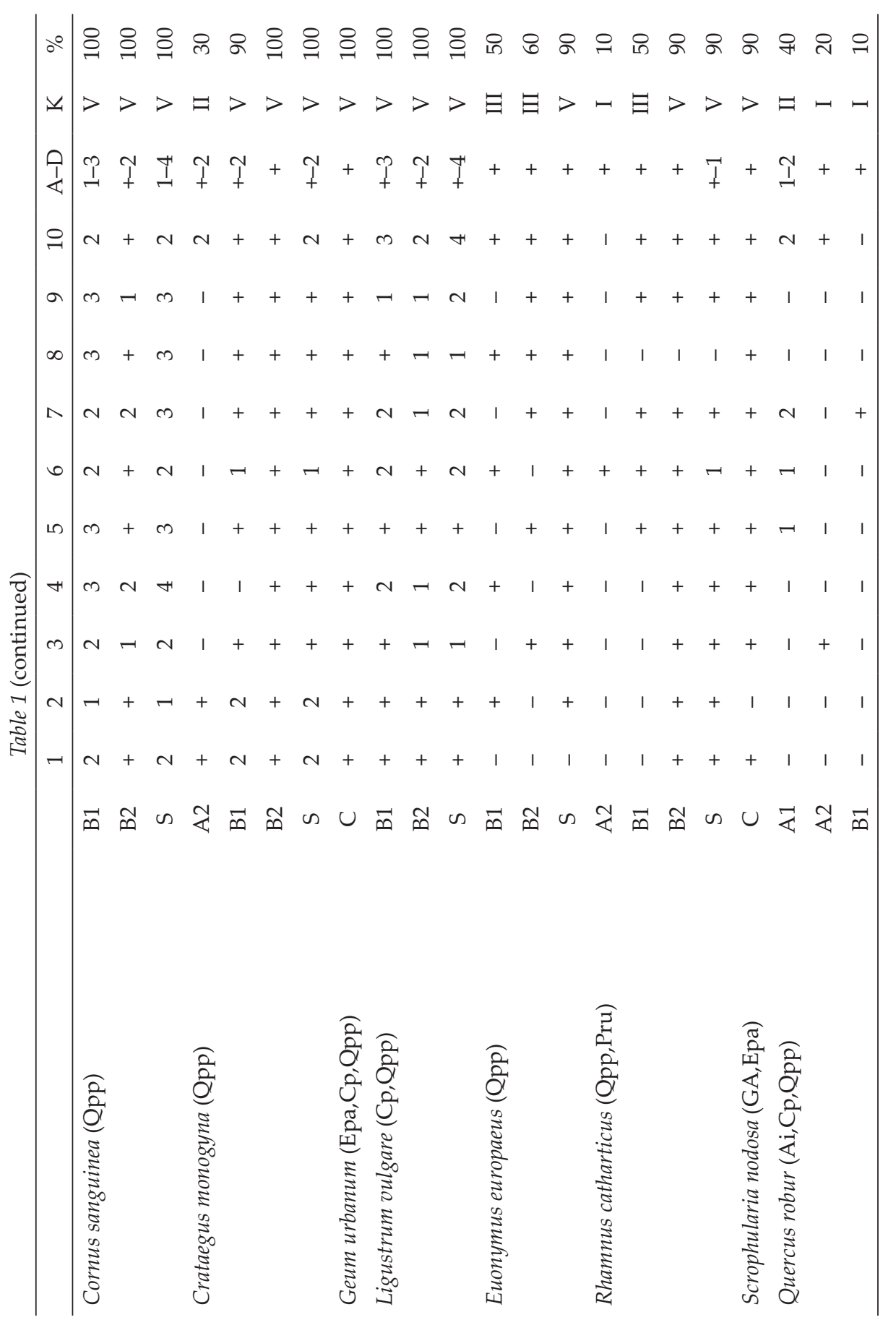




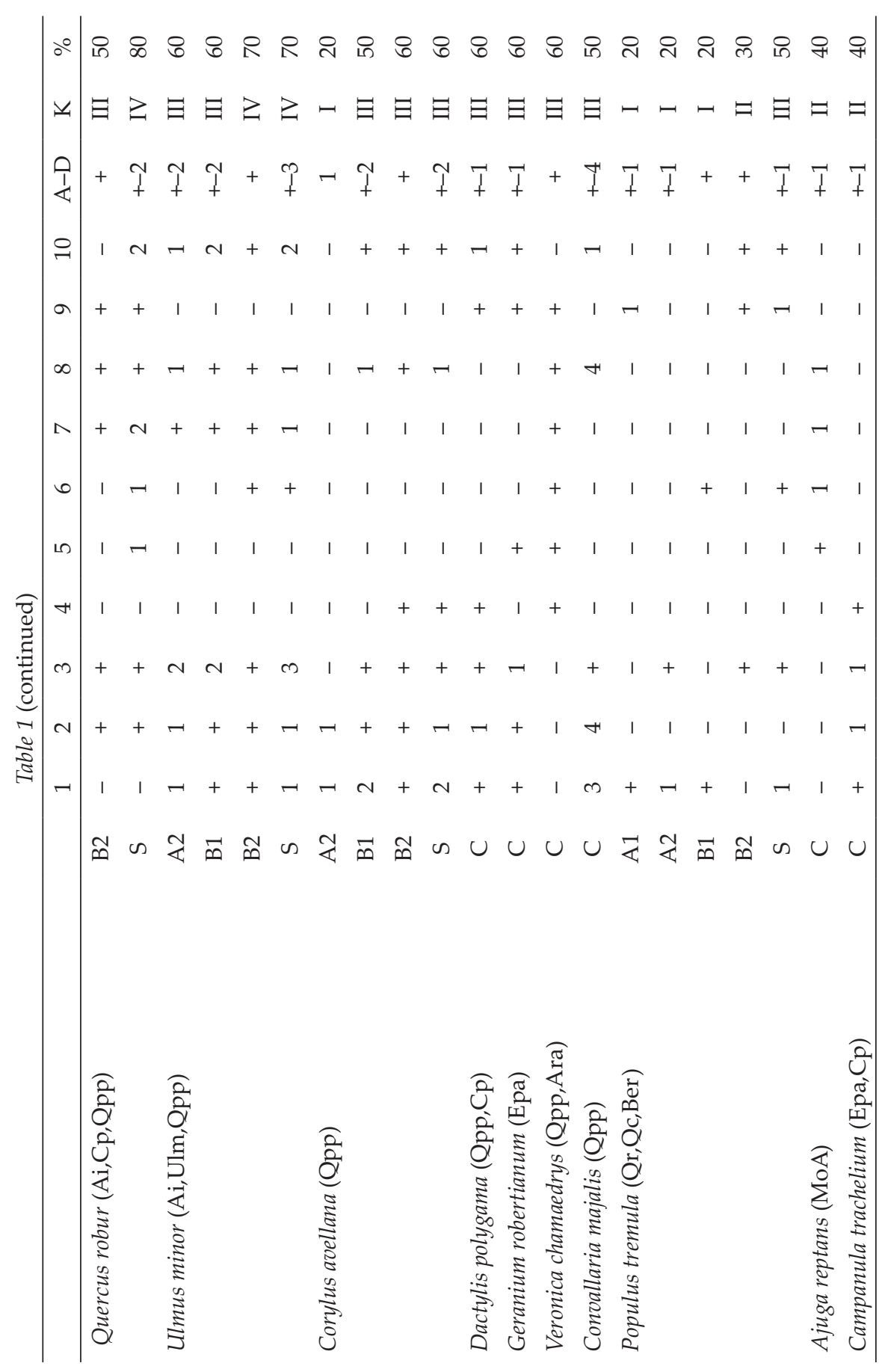




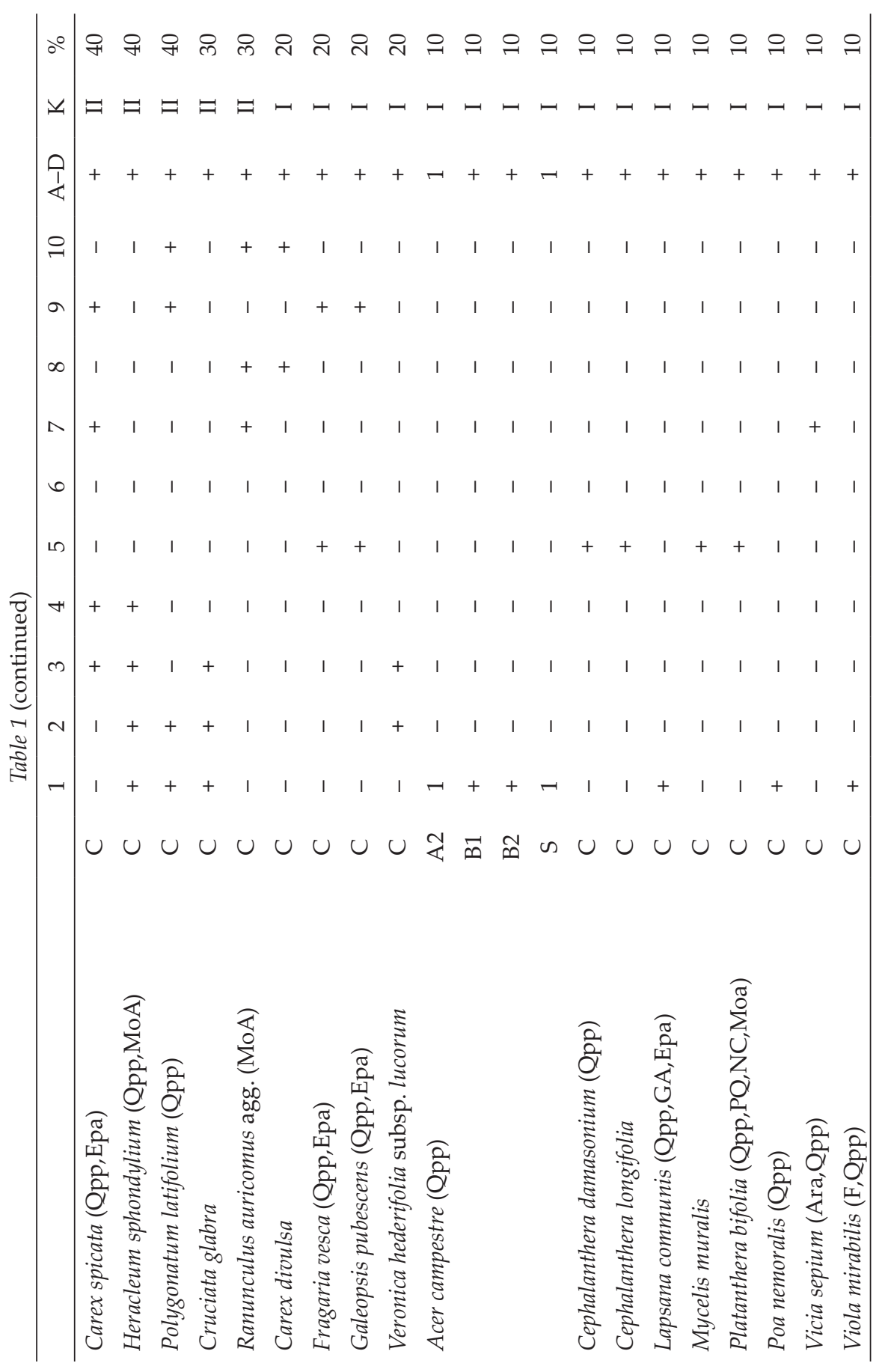




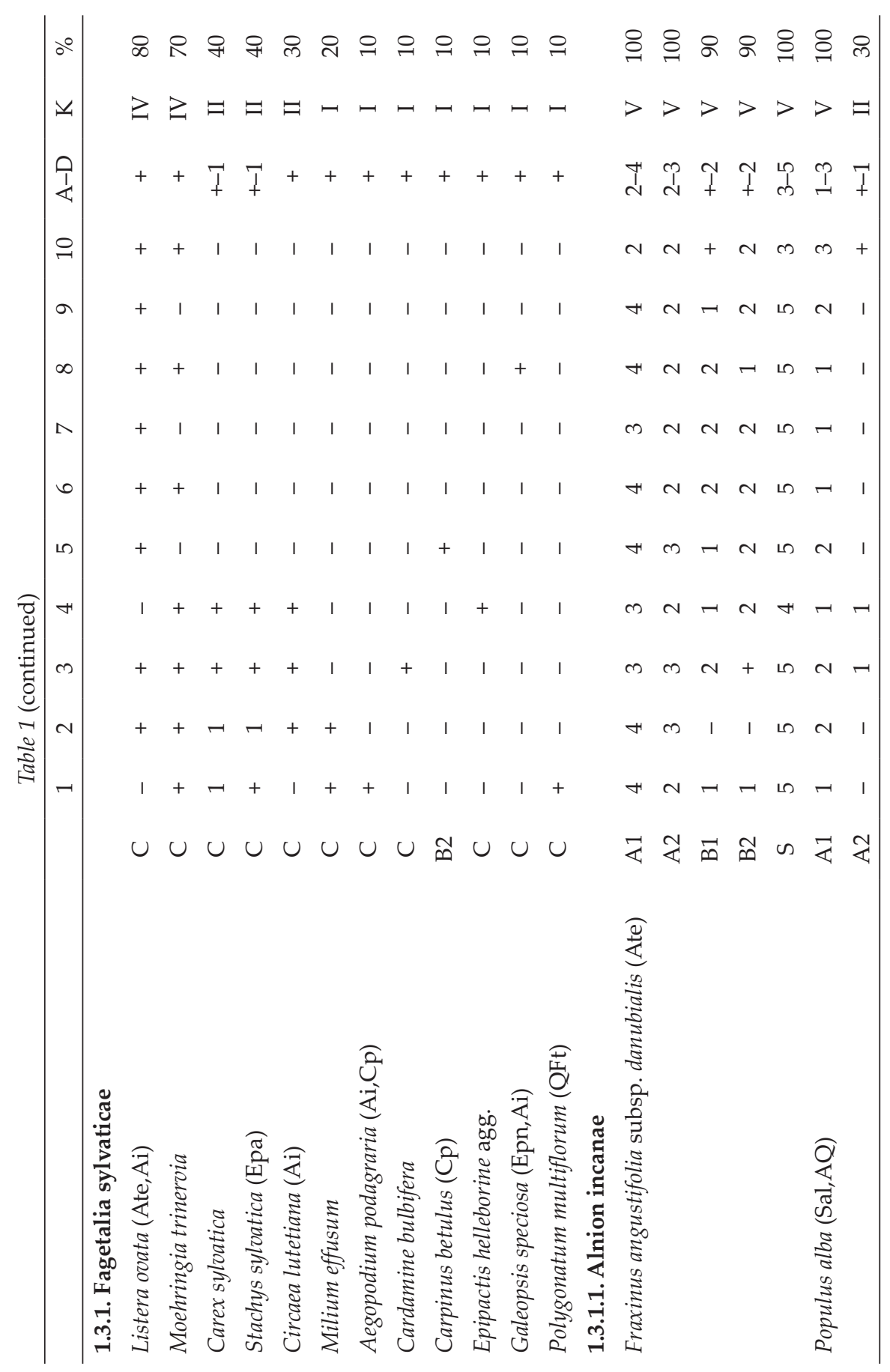




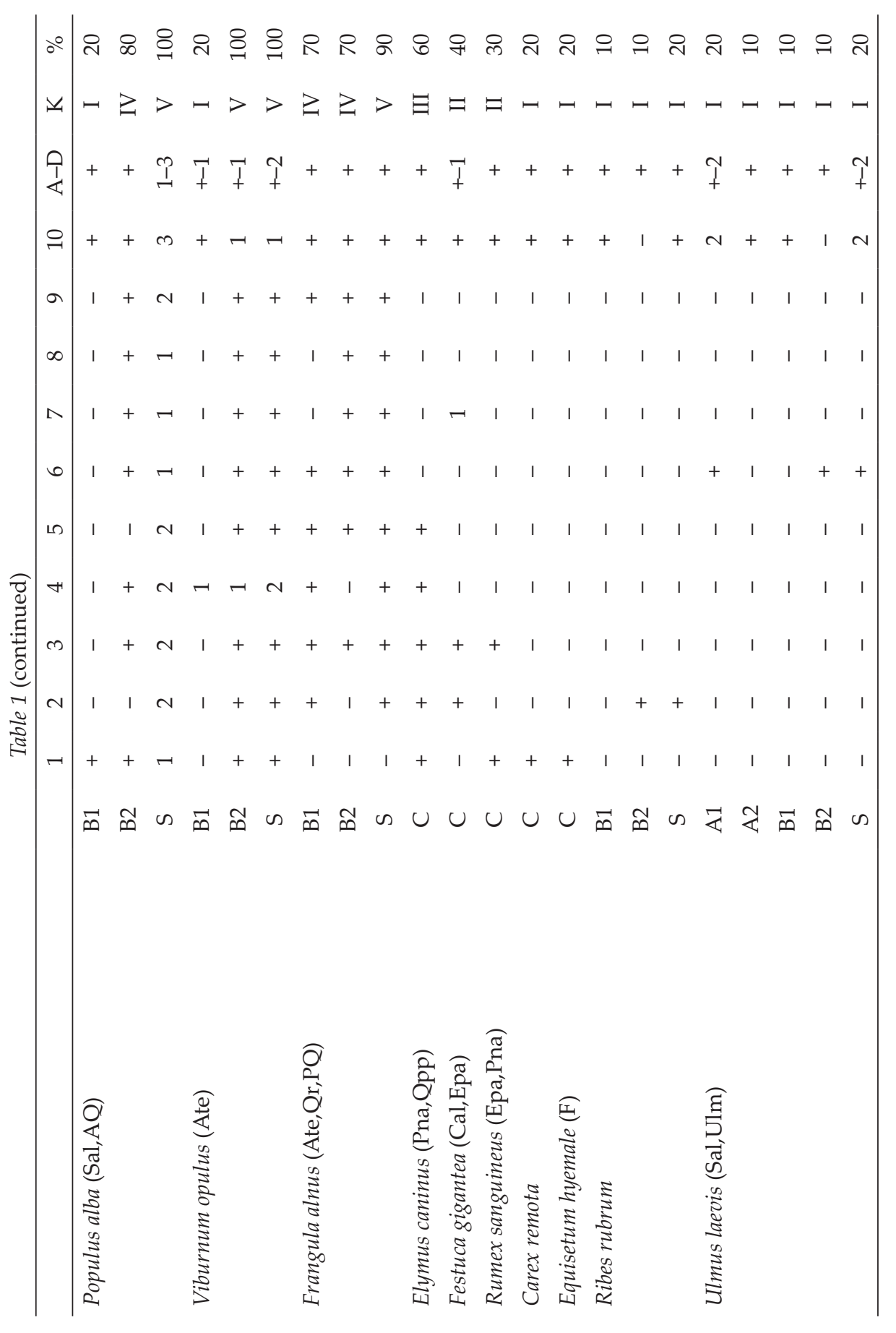




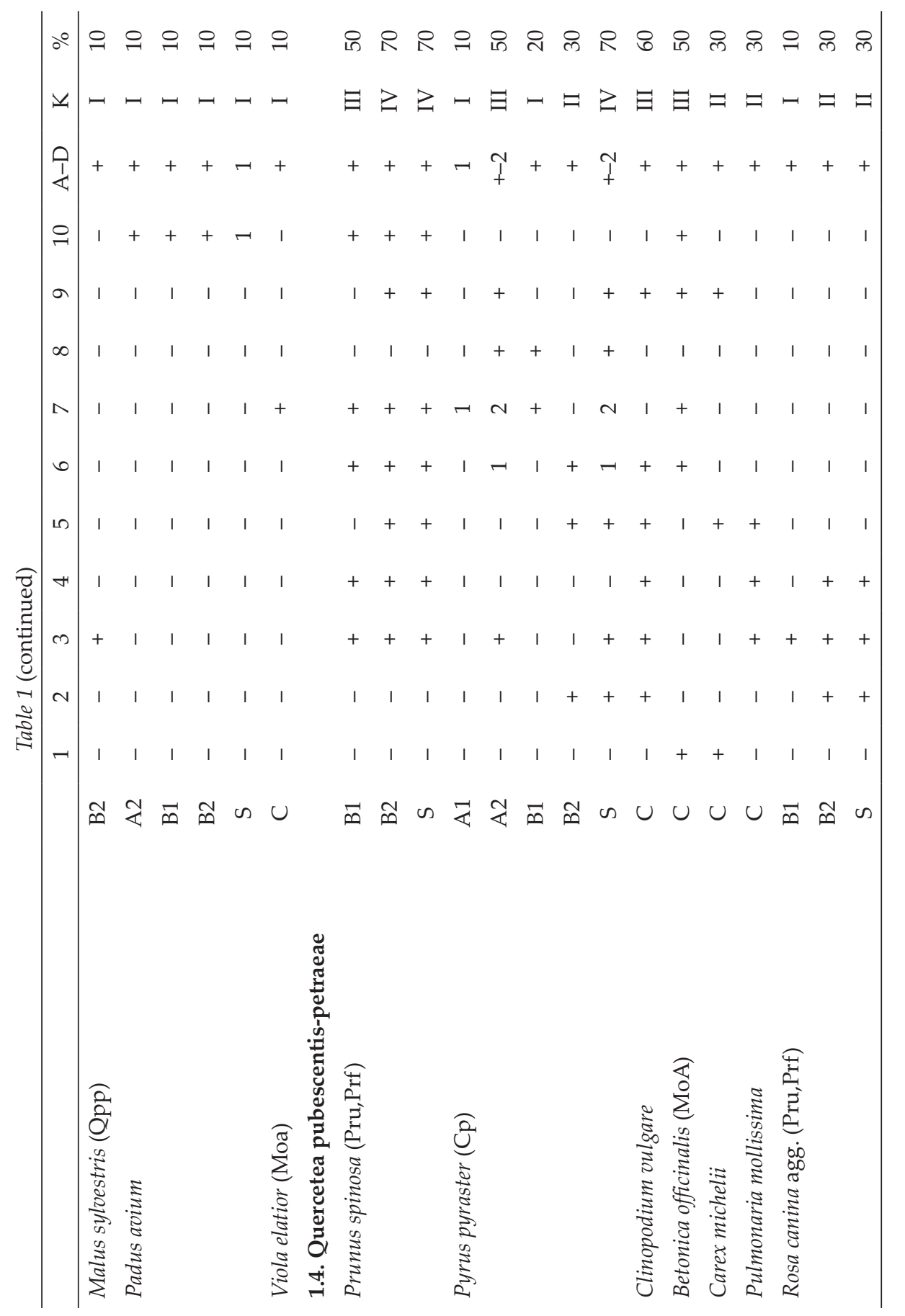




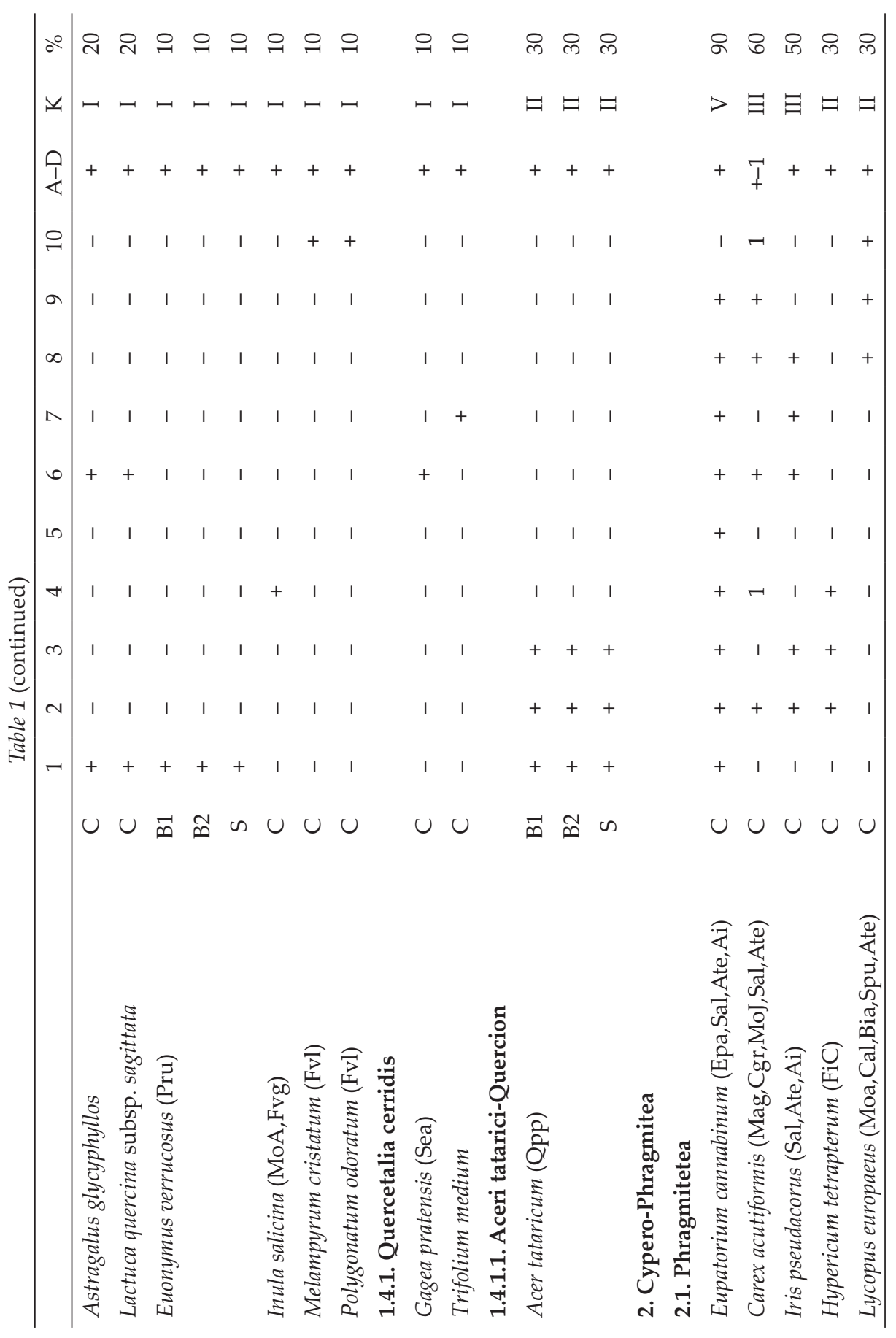




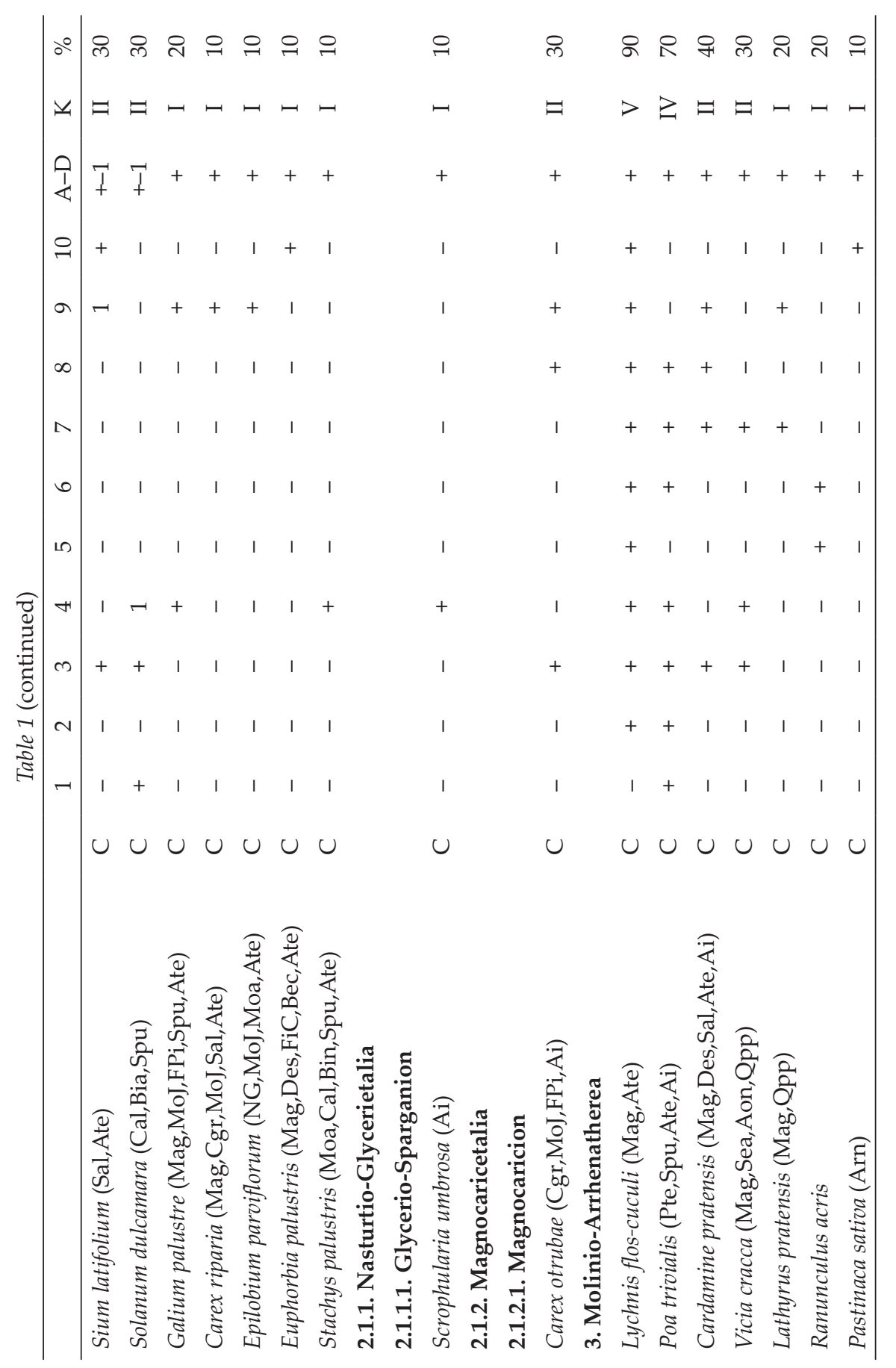




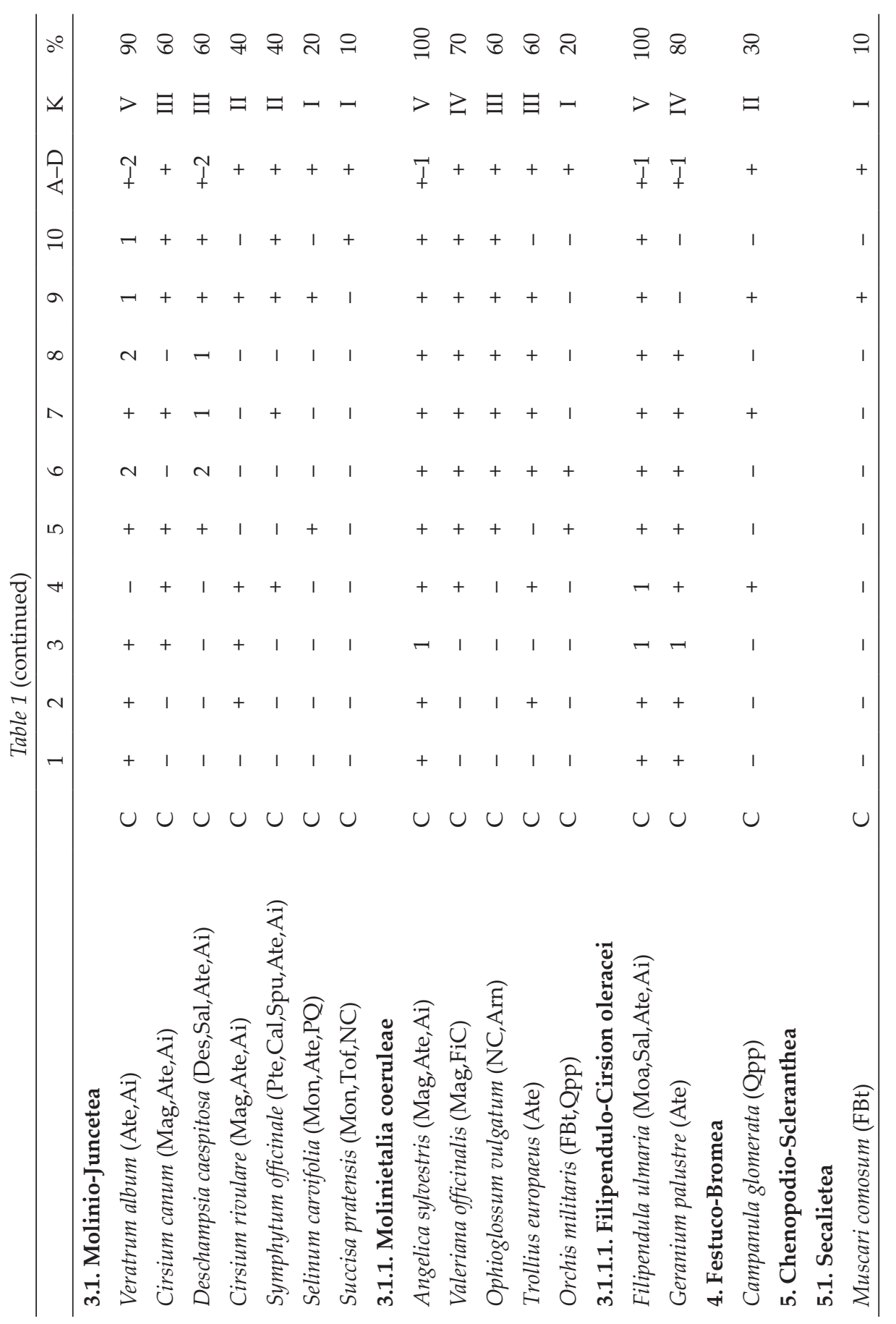




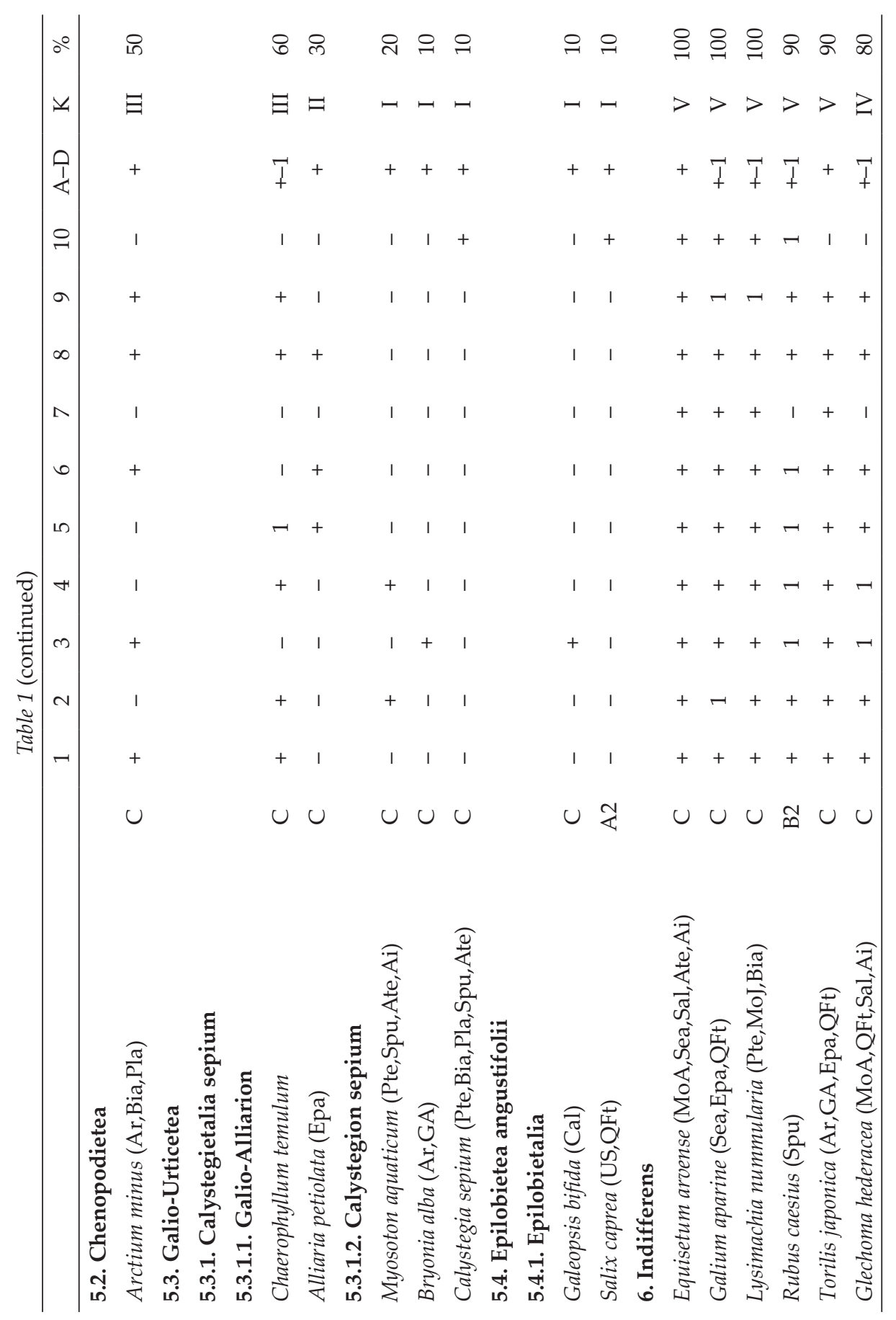




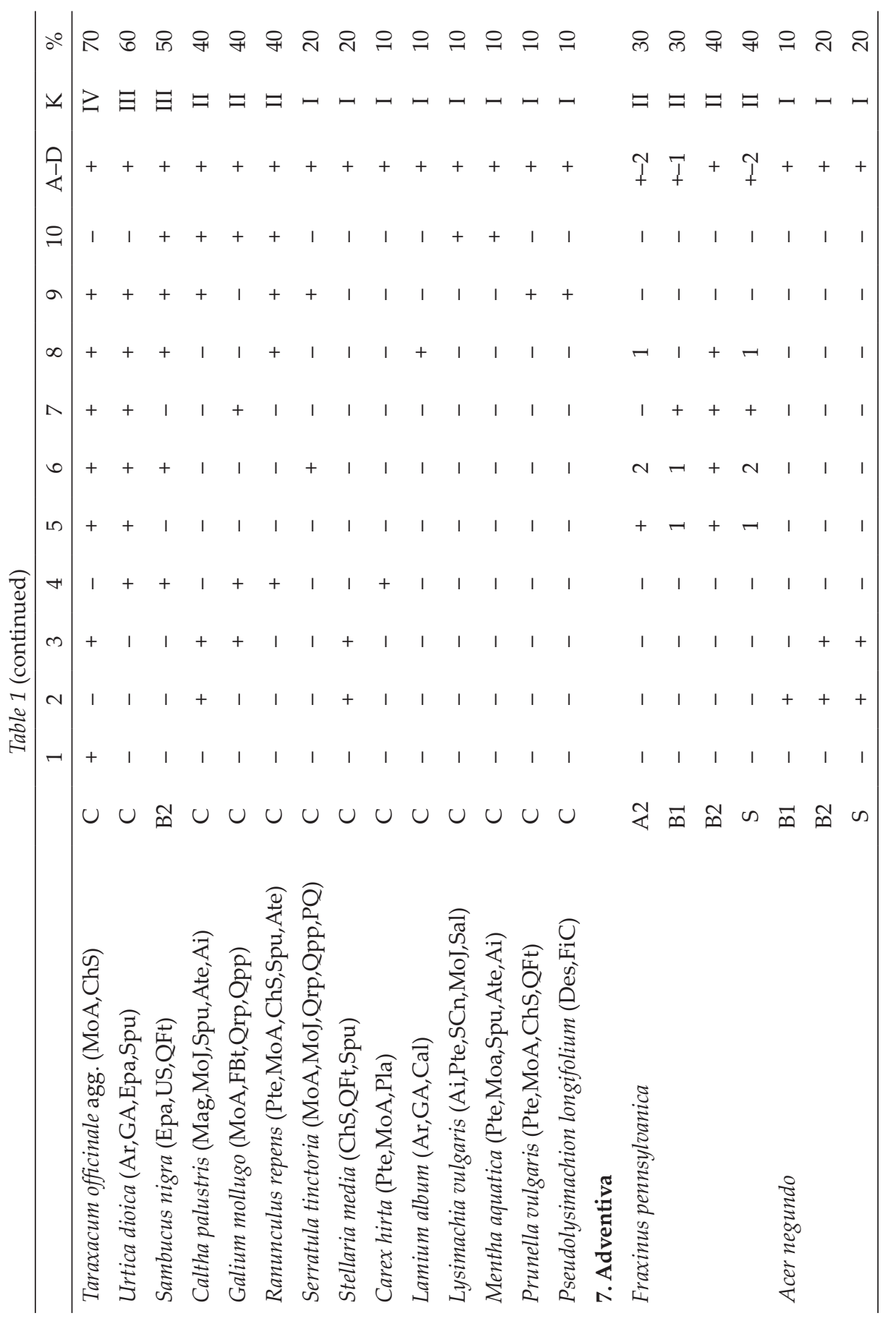




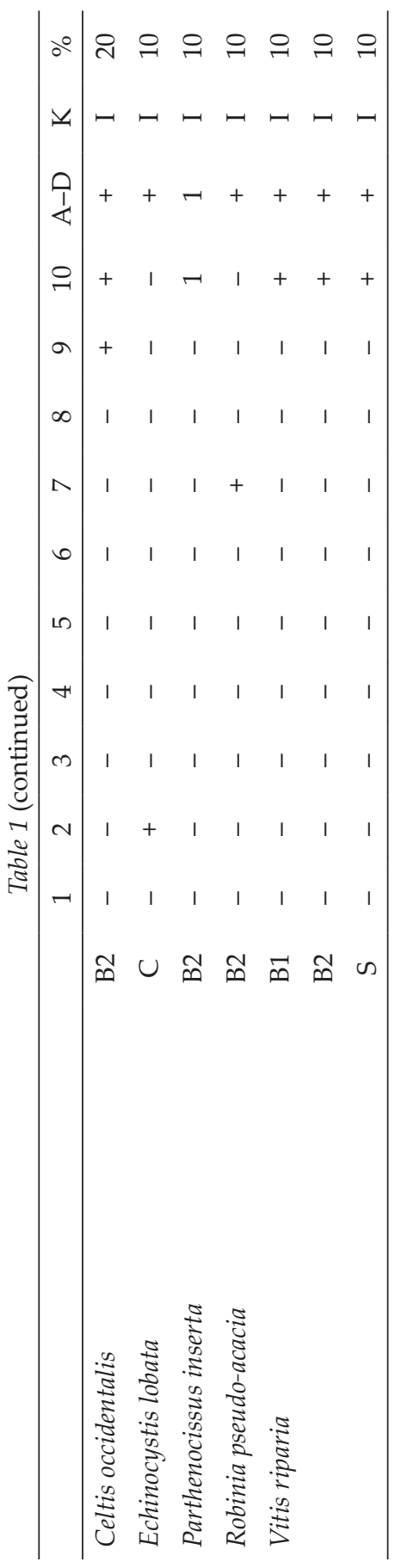

Frequency distribution of constancy classes

The ten samples included 22 constant (K: $\mathrm{V})$ and 12 sub-constant $\mathrm{K}: \mathrm{IV})$ species. The number of accessorial (K: III), sub-accessorial (K: II), and accidental (K: I) species in the samples was 19, 37, and 69, respectively (see Table 1).

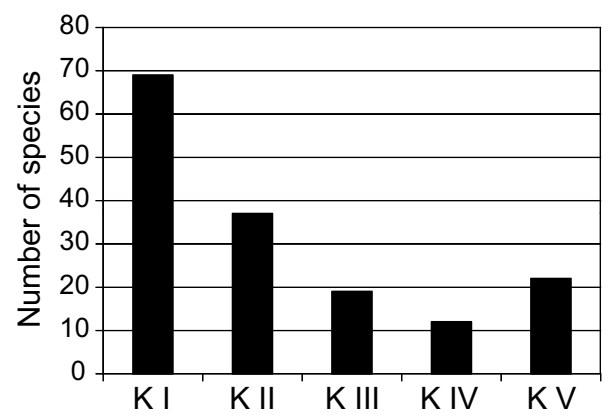

Fig. 4. Frequency distribution of species in different constancy classes in Veratro albiFraxinetum angustifoliae

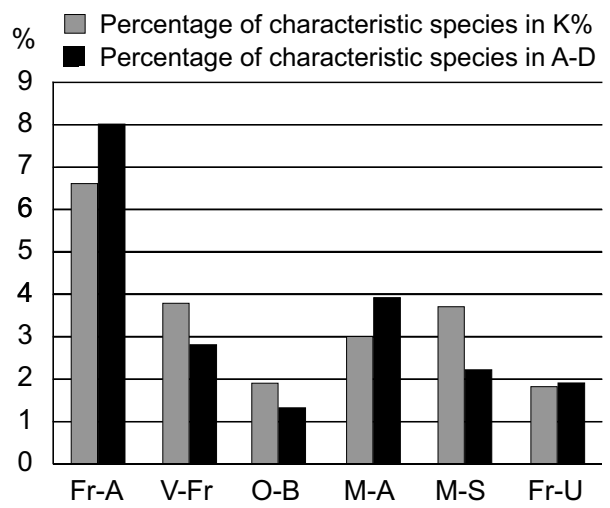

Fig. 5. Proportion of Alnetea glutinosae s. 1. character species. $\mathrm{Fr}-\mathrm{A}=$ Fraxino pannonicae-Alnetum, Nyírség (Kevey and Papp L. ined.: 5 relevés); $\mathrm{V}-\mathrm{Fr}=$ Veratro albi-Fraxinetum angustifoliae, Nyírség (Kevey and Papp L. ined.: 10 relevés); Fr-U = Fraxino pannonicae-Ulmetum, Nyírség (Kevey et al. 2017: 20 relevés) 


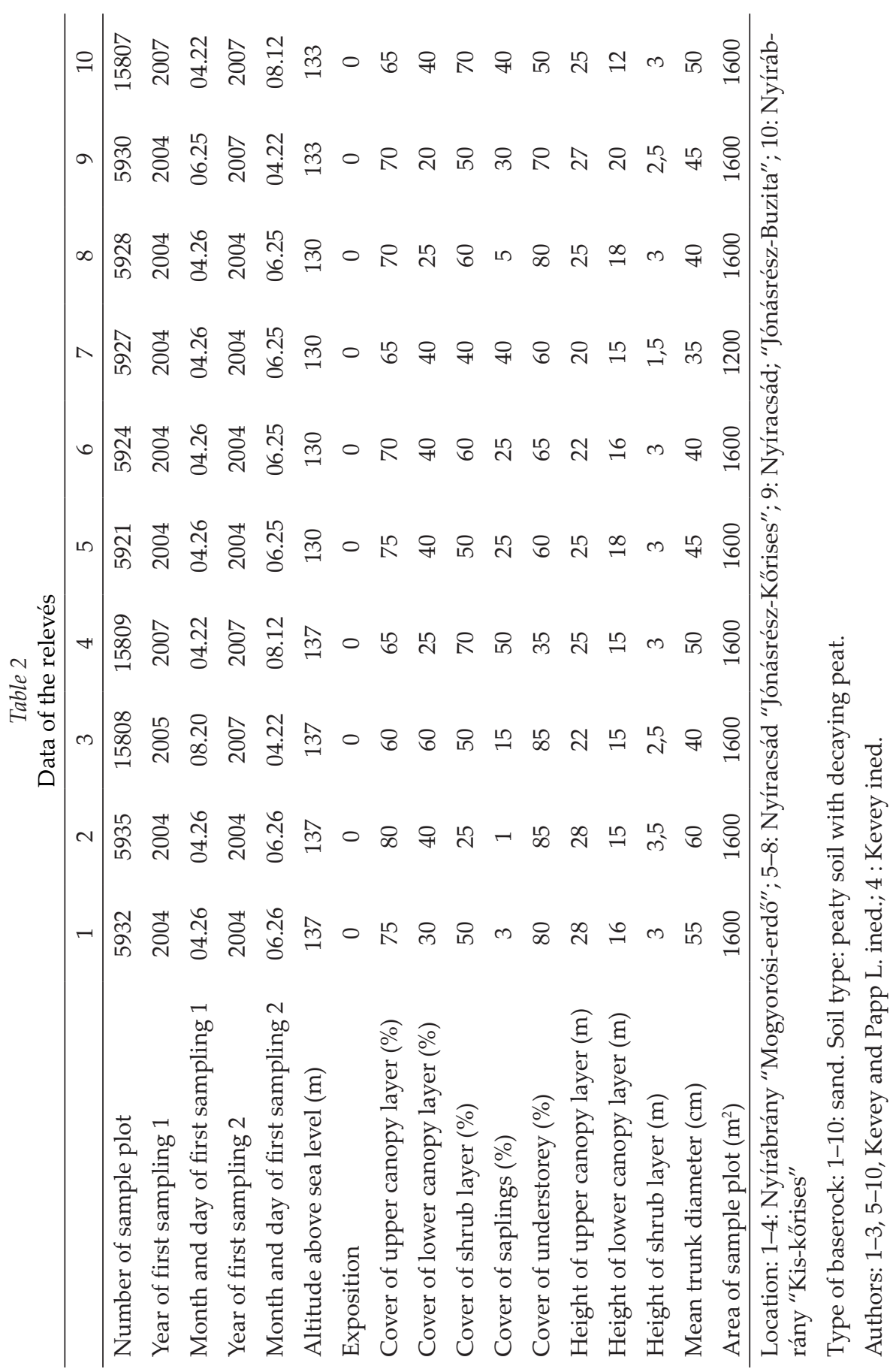




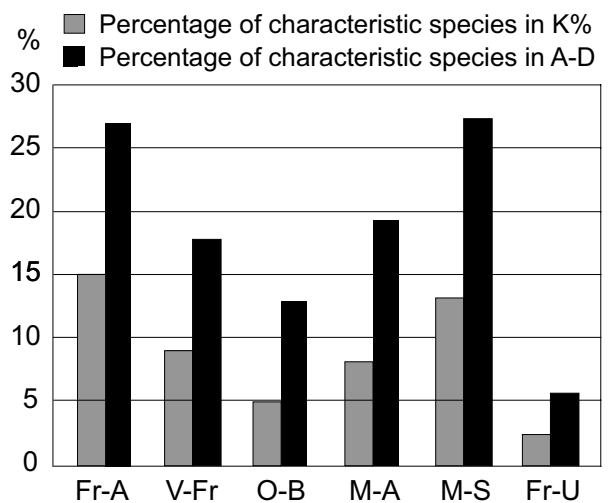

Fig. 6. Proportion of Salicetea purpureae s. 1. character species. Fr-A = Fraxino pannonicae-Alnetum, Nyírség (Kevey and Papp L. ined.: 5 relevés); $\mathrm{V}-\mathrm{Fr}=$ Veratro albi-Fraxinetum angustifoliae, Nyírség (Kevey and Papp L. ined.: 10 relevés); Fr-U = Fraxino pannonicae-Ulmetum, Nyírség (Kevey et al. 2017: 20 relevés)

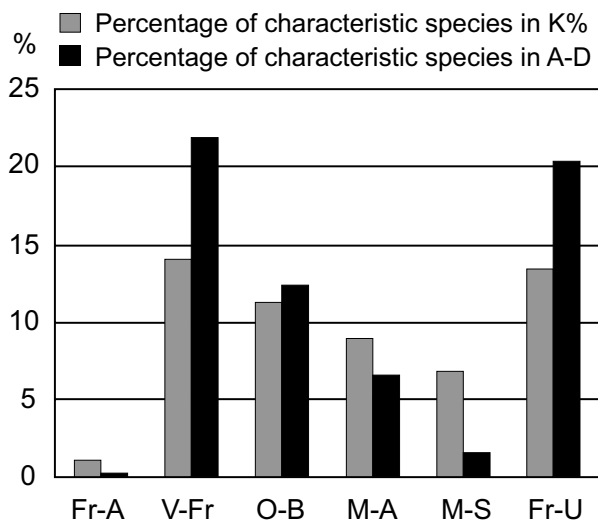

Fig. 8. Proportion of Fagetalia character species. Fr-A = Fraxino pannonicae-Alnetum, Nyírség (Kevey and Papp L. ined.: 5 relevés); V-Fr = Veratro albi-Fraxinetum angustifoliae, Nyírség (Kevey and Papp L. ined.: 10 relevés); Fr-U = Fraxino pannonicae-Ulmetum, Nyírség (Kevey et al. 2017: 20 relevés)

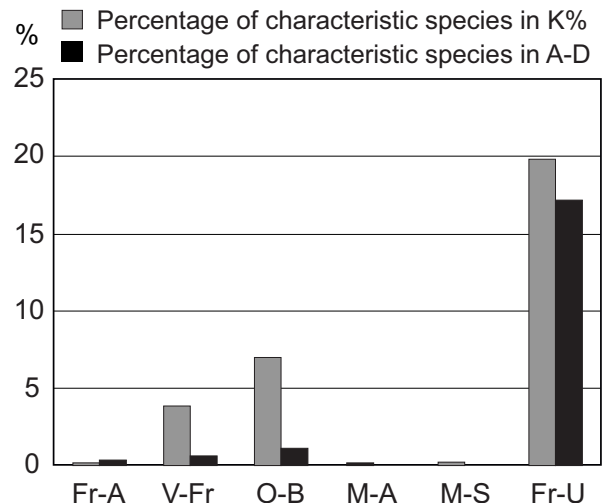

Fig. 7. Proportion of Alnion incanae s. 1. character species. Fr-A = Fraxino pannonicae-Alnetum, Nyírség (Kevey and Papp L. ined.: 5 relevés); $\mathrm{V}-\mathrm{Fr}=$ Veratro albi-Fraxinetum angustifoliae, Nyírség (Kevey and Papp L. ined.: 10 relevés); Fr-U = Fraxino pannonicae-Ulmetum, Nyírség (Kevey et al. 2017: 20 relevés)

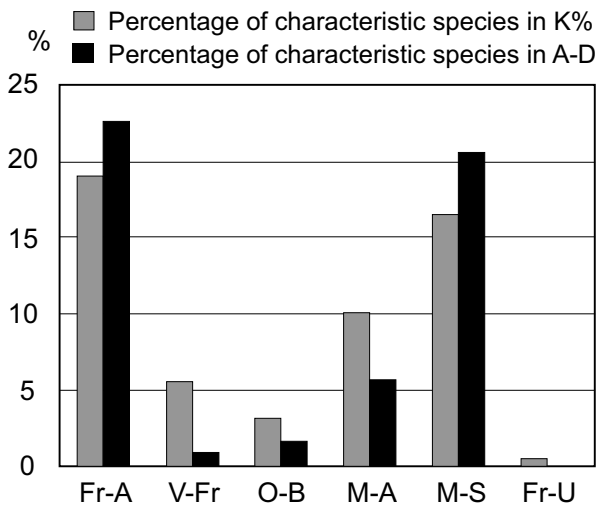

Fig. 9. Proportion of Phragmitetea s. 1. character species. Fr-A = Fraxino pannonicae- $\mathrm{Al}$ netum, Nyírség (Kevey and Papp L. ined.: 5 relevés); $\mathrm{V}-\mathrm{Fr}=$ Veratro albi-Fraxinetum angustifoliae, Nyírség (Kevey and Papp L. ined.: 10 relevés); Fr-U = Fraxino pannonicae-Ulmetum, Nyírség (Kevey et al. 2017: 20 relevés) 


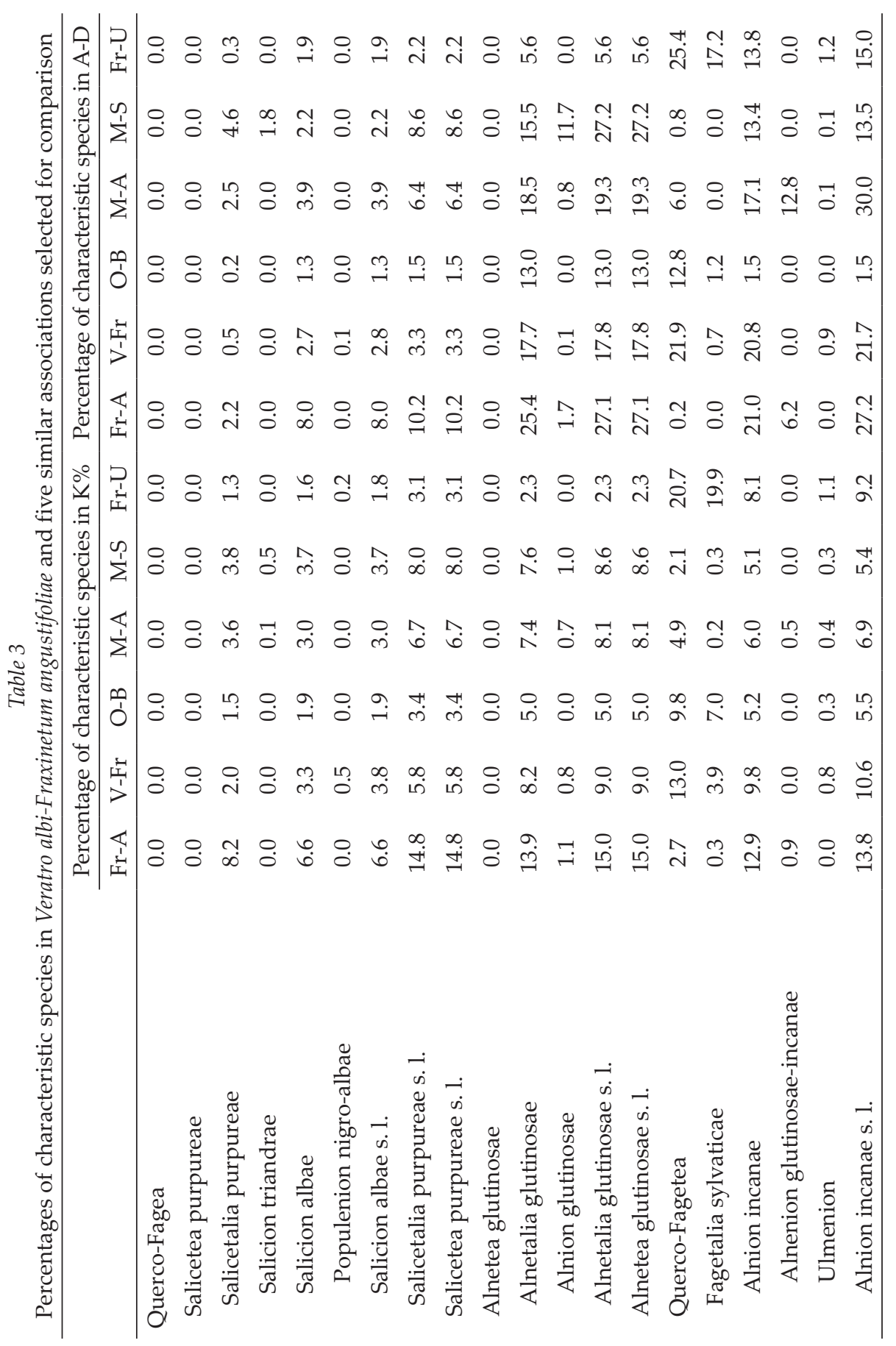




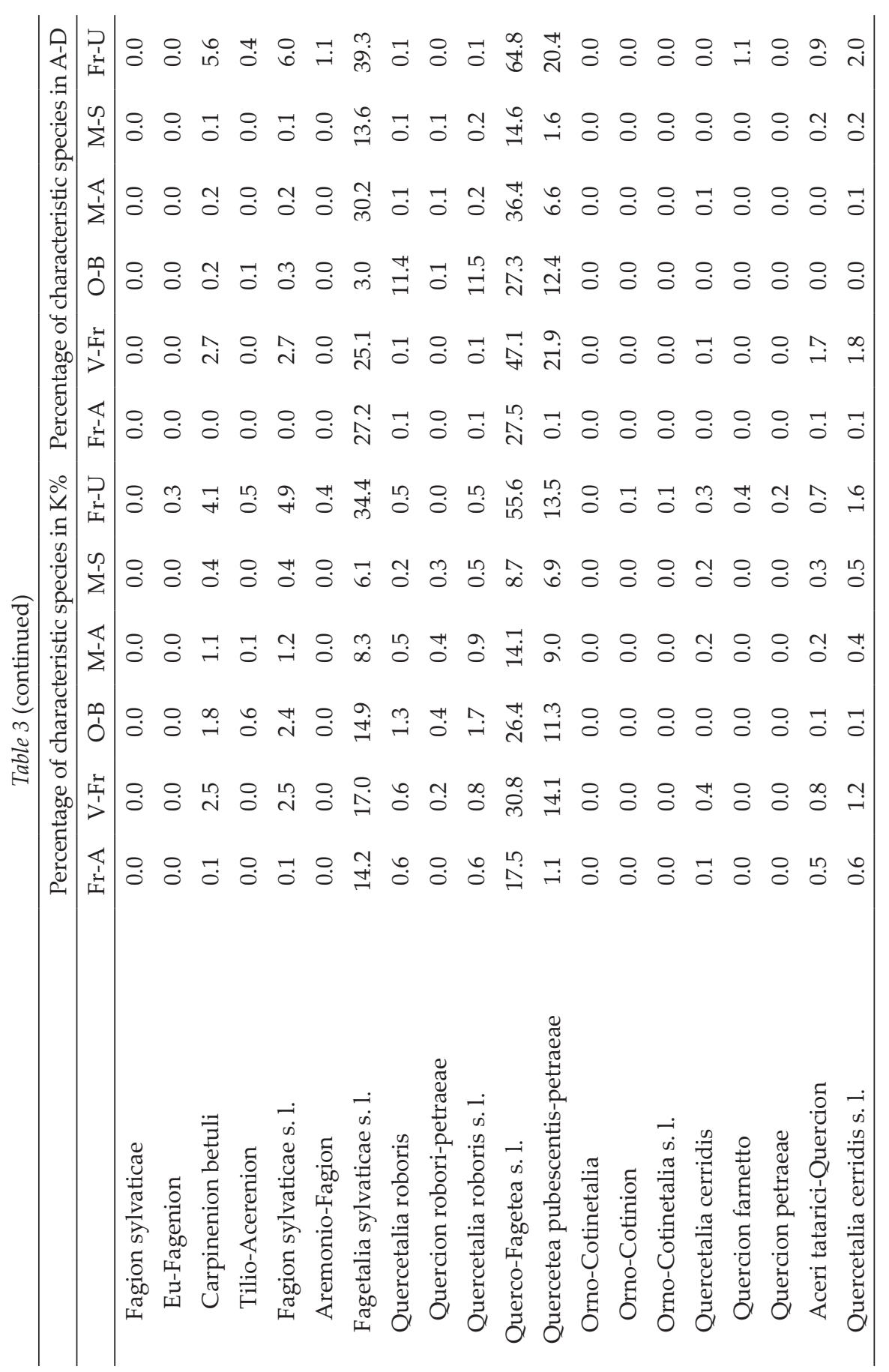




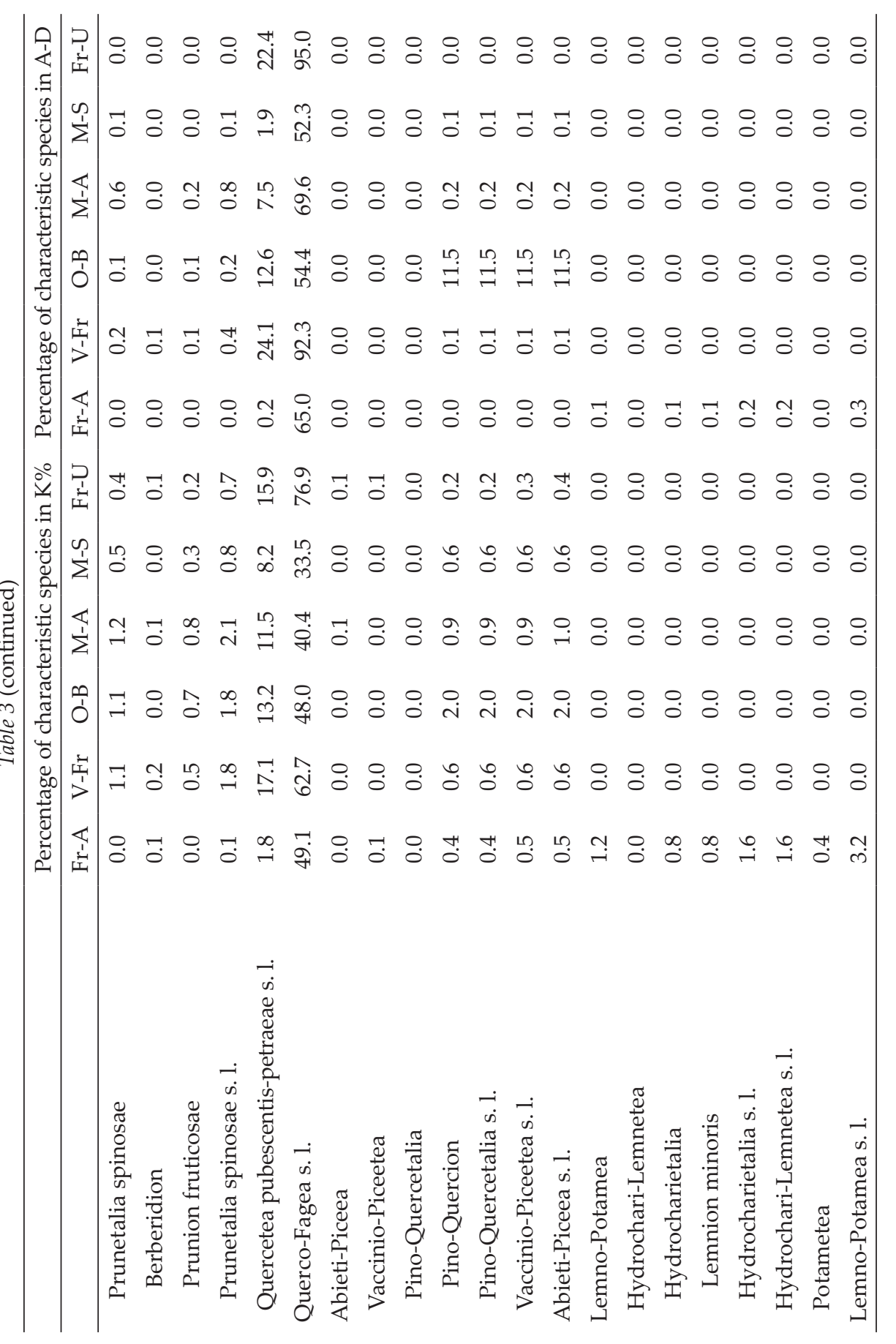




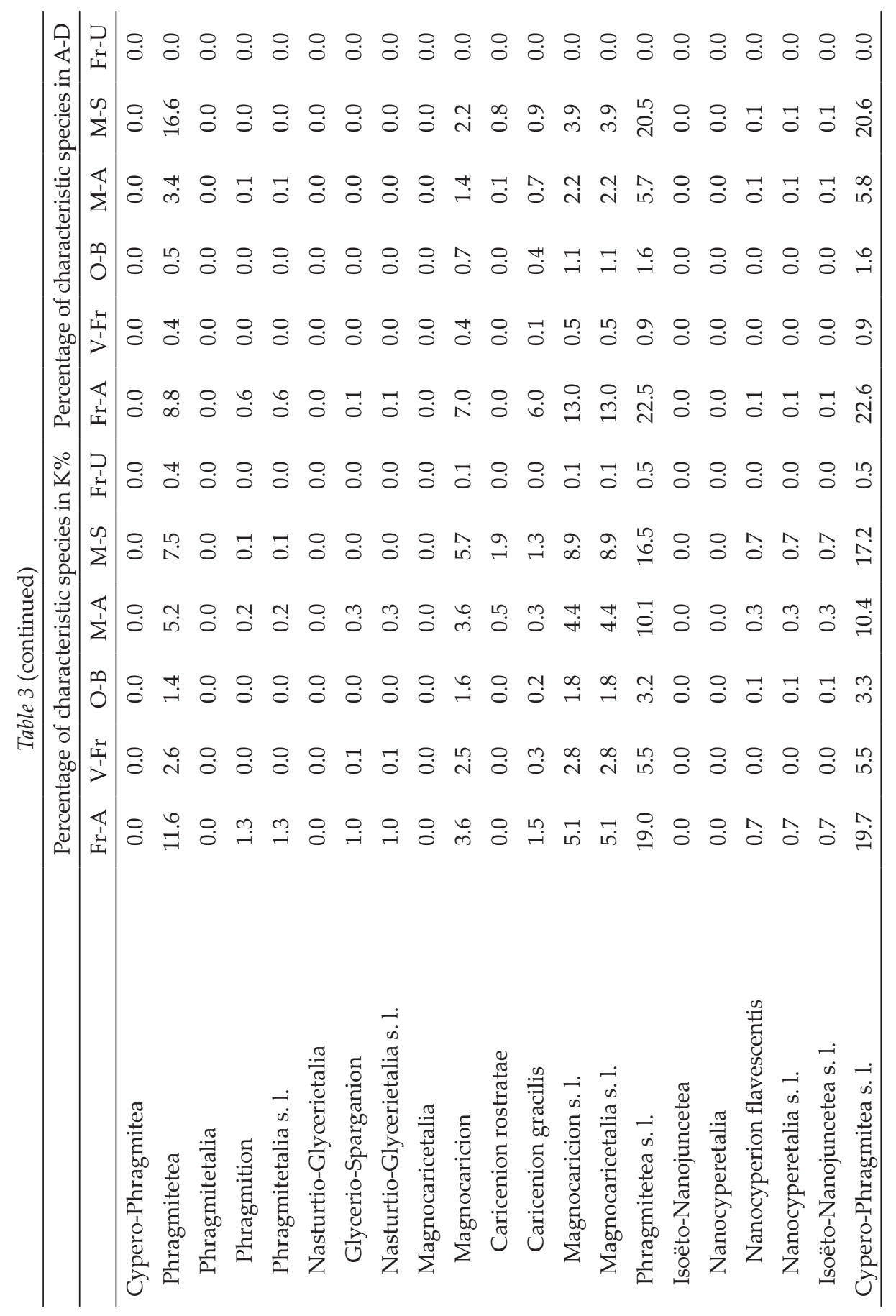




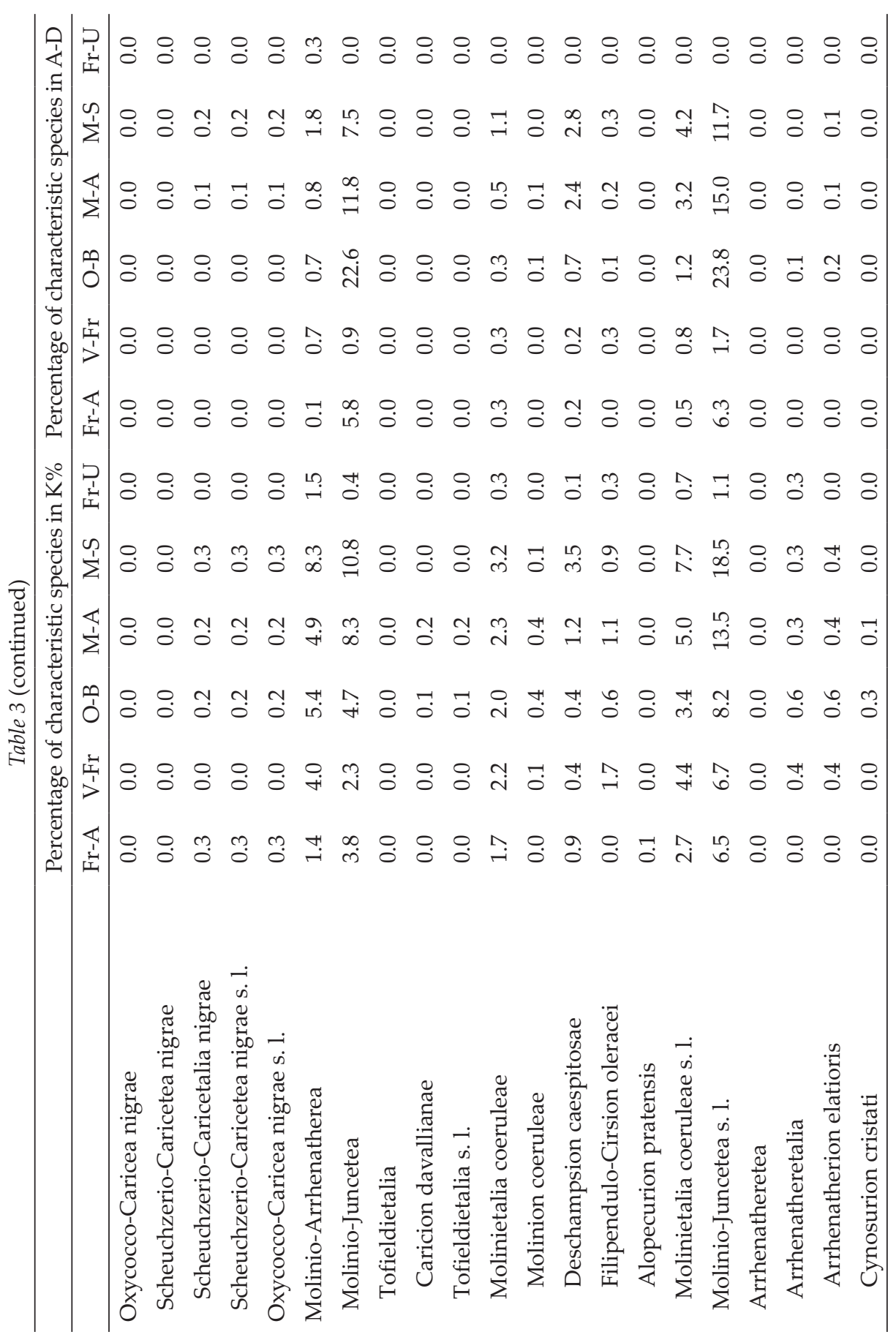




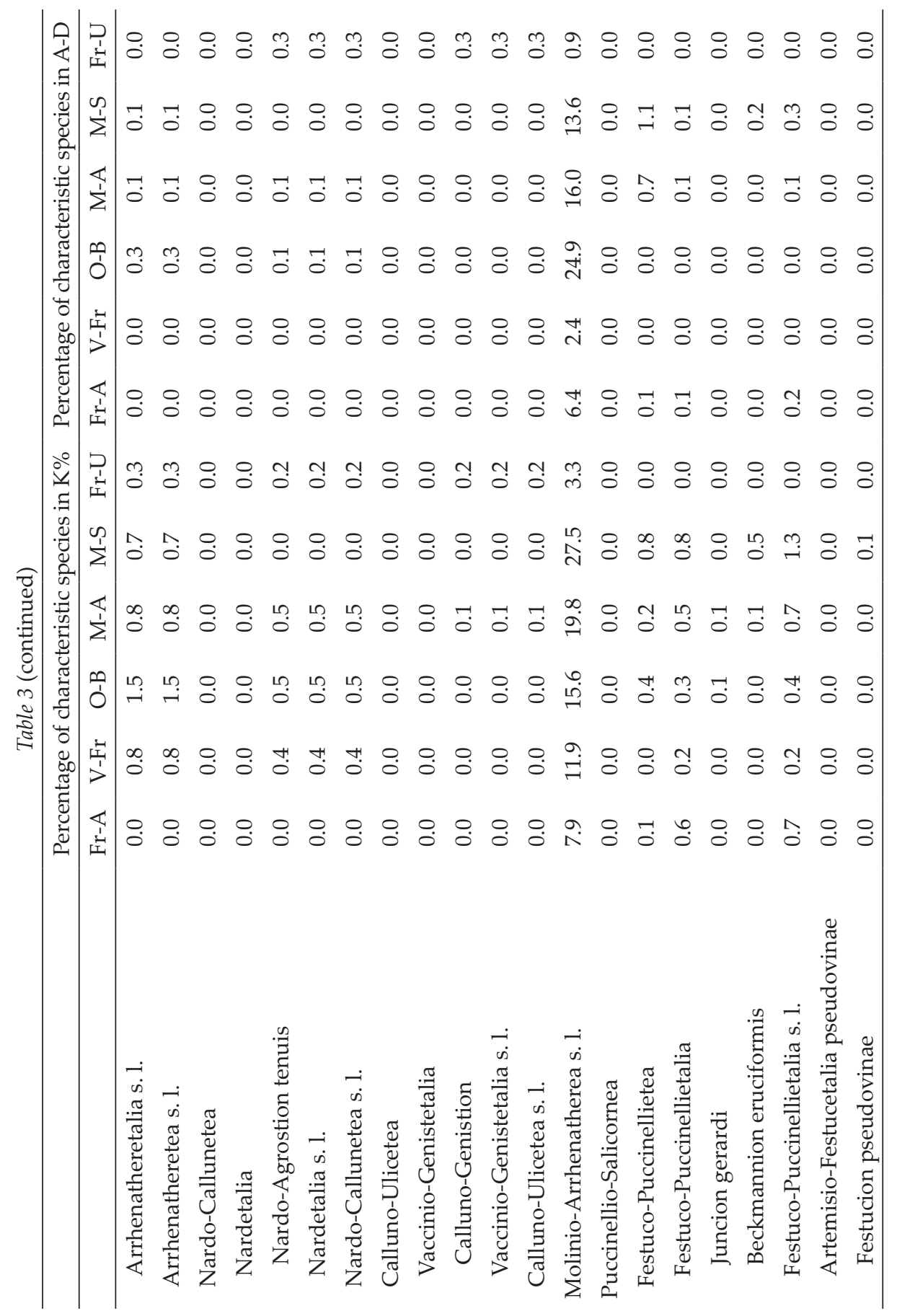




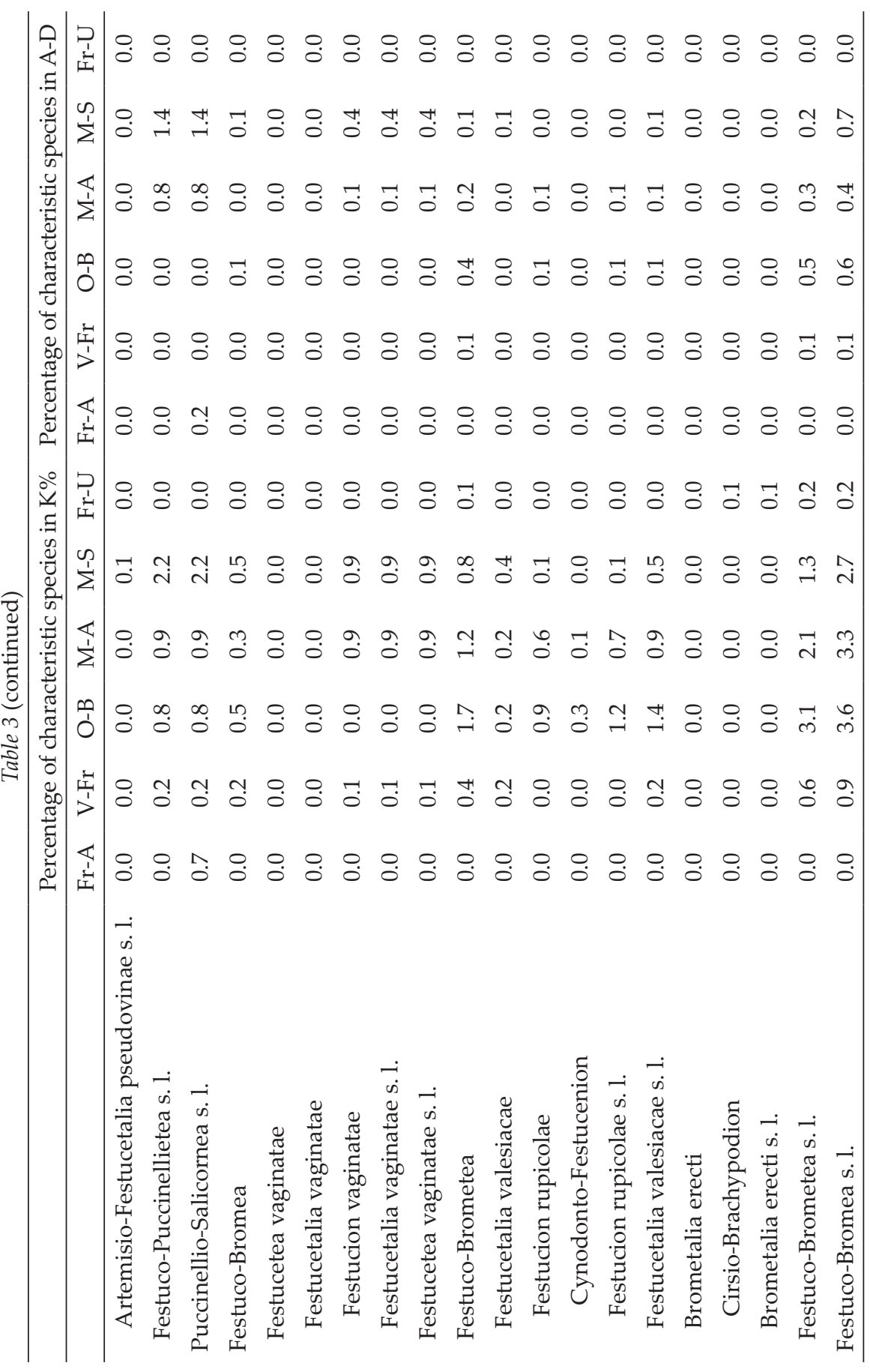




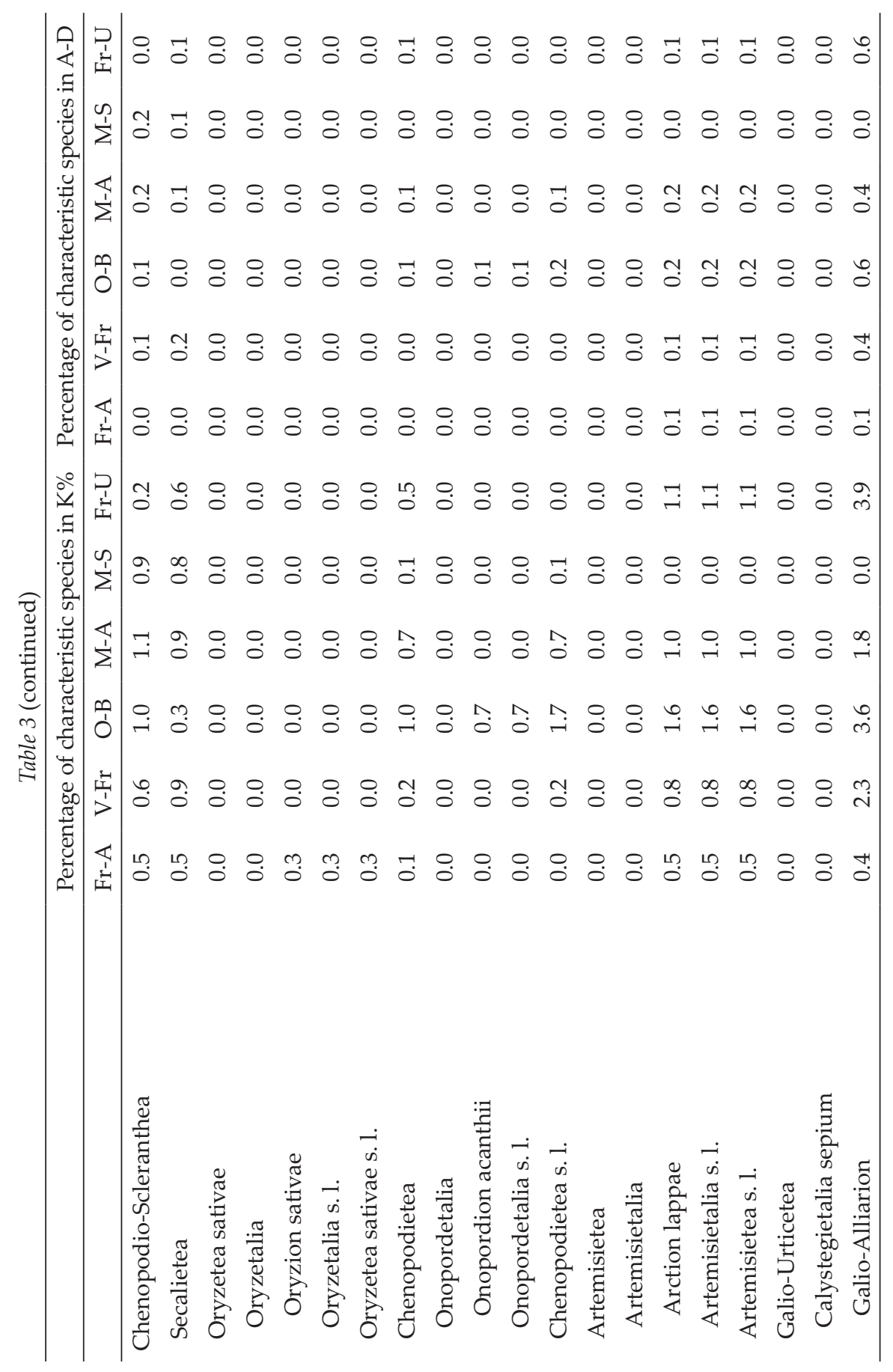




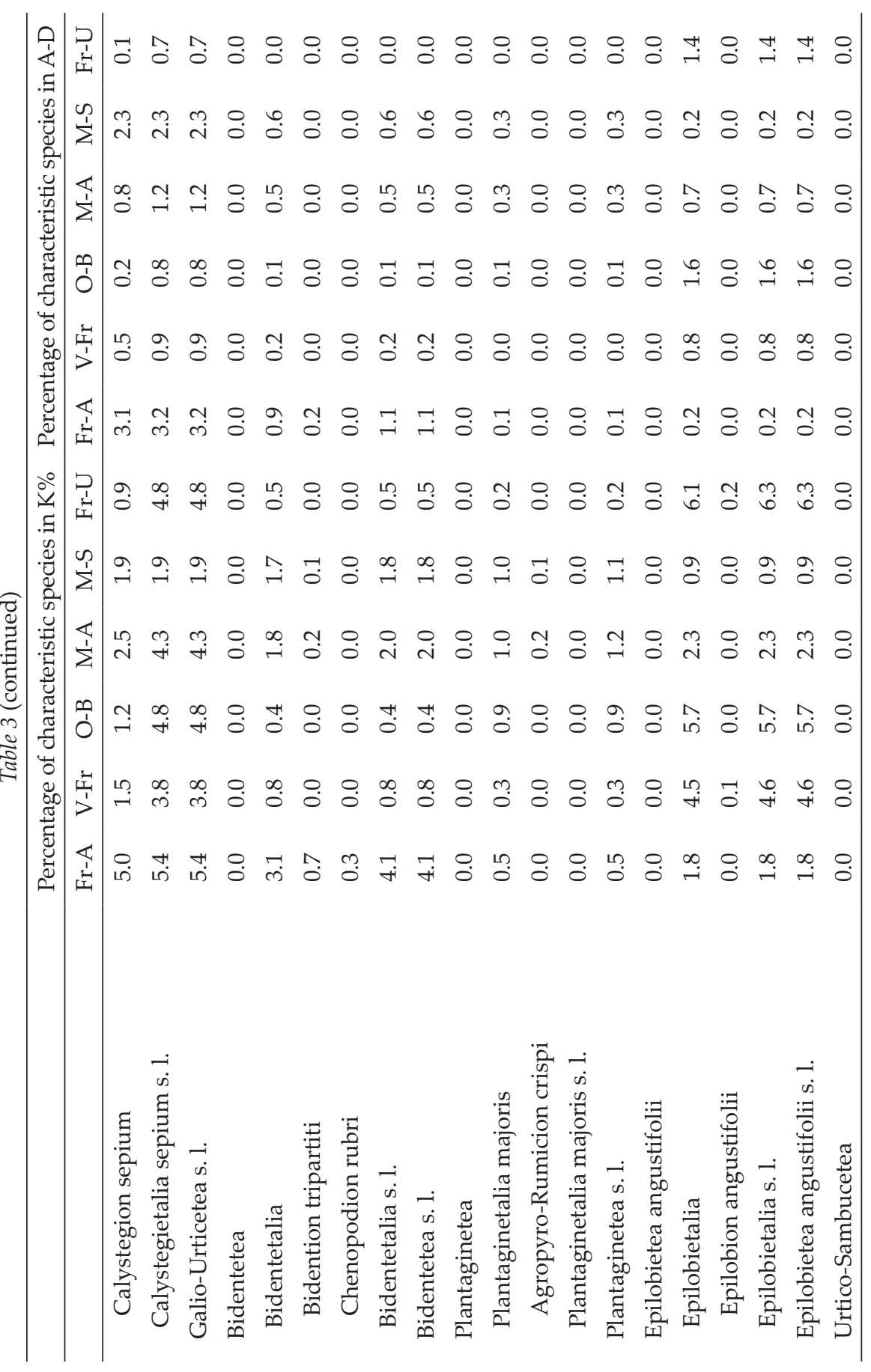




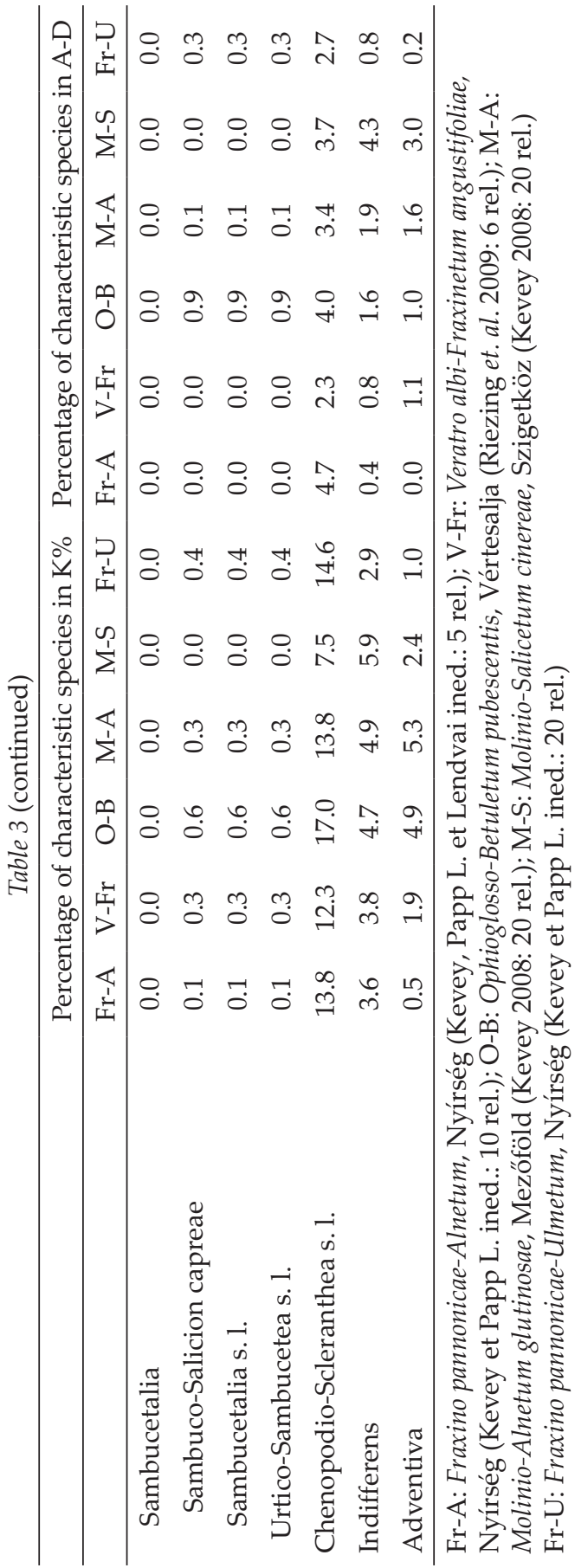

The frequency distribution of species in the five constancy categories, from I to $\mathrm{V}$, follows a characteristic pattern. The highest frequency value at I steeply drops to II, then decreases further with diminishing increments to $\mathrm{IV}$, where it reaches its minimum. At $V$, its value is again higher (Fig. 4). This patterns is only typical of phytosociological samples that are representative of the sampled vegetation unit (see Kevey 2008).

\section{Proportion of character species}

As usual in temperate deciduous forests in Central Europe, the species characteristic of the Querco-Fagetea class or syntaxa within it play a substantial role $(30.8 \%)$ in the species composition of these Fraxinus angustifolia dominated stands. They are followed by the character species of SE European dry oak woods, Quercetea pubescentis-petraeae s. 1. $(17.1 \%)$ despite the rather mesic habitat, and Alnetea glutinosae s. 1., Molinio-Juncetea s. 1., Salicetea s. 1., and Phragmitetea s. 1. in decreasing order. Within the Querco-Fagetea class, Fagetalia s. 1. and Alnion incanae species attain the highest proportions.

In comparison to other communities, it is noteworthy that character species proportions in all but two syntaxa were typically intermediate between those in Fraxino pannonicae-Alnetum and 
Table 4

Differential species in Veratro albi-Fraxinetum angustifoliae and Fraxino pannonicae-Alnetum

\begin{tabular}{|c|c|c|c|c|c|}
\hline & $\mathrm{V}-\mathrm{Fr}$ & Fr-A & & V-Fr & Fr-A \\
\hline Constant species & & & Geranium palustre & IV & - \\
\hline Crataegus monogyna & $\mathrm{V}$ & - & Moehringia trinervia & IV & - \\
\hline Euonymus europaeus & $\mathrm{V}$ & - & Prunus spinosa & IV & - \\
\hline Filipendula ulmaria & $\mathrm{V}$ & - & Pyrus pyraster & IV & - \\
\hline Geum urbanum & $\mathrm{V}$ & - & Taraxacum officinale agg. & IV & - \\
\hline Ligustrum vulgare & $\mathrm{V}$ & - & Ulmus minor & IV & - \\
\hline Lychnis flos-cuculi & $\mathrm{V}$ & - & Valeriana officinalis & IV & - \\
\hline Rhamnus catharticus & $\mathrm{V}$ & - & Listera ovata & IV & I \\
\hline Scrophularia nodosa & $\mathrm{V}$ & - & Quercus robur & IV & I \\
\hline Torilis japonica & $\mathrm{V}$ & - & Glechoma hederacea & IV & II \\
\hline Veratrum album & $\mathrm{V}$ & - & Alisma plantago-aquatica & - & IV \\
\hline Angelica sylvestris & $\mathrm{V}$ & I & Sparganium erectum & - & IV \\
\hline Equisetum arvense & $\mathrm{V}$ & I & Calystegia sepium & I & IV \\
\hline Galium aparine & $\mathrm{V}$ & I & Lysimachia vulgaris & I & IV \\
\hline Brachypodium sylvaticum & $\mathrm{V}$ & II & Mentha aquatica & I & IV \\
\hline Cornus sanguinea & $\mathrm{V}$ & II & Stachys palustris & I & IV \\
\hline Lysimachia nummularia & $\mathrm{V}$ & II & Sium latifolium & II & IV \\
\hline Viburnum opulus & $\mathrm{V}$ & II & Accessorial species & & \\
\hline Frangula alnus & $\mathrm{V}$ & III & Betonica officinalis & III & - \\
\hline Populus alba & $\mathrm{V}$ & III & Chaerophyllum temulum & III & - \\
\hline Alnus glutinosa & - & $\mathrm{V}$ & Cirsium canum & III & - \\
\hline Lemna minor & - & $\mathrm{V}$ & Clinopodium vulgare & III & - \\
\hline Oenanthe aquatica & - & $\mathrm{V}$ & Convallaria majalis & III & - \\
\hline Salix cinerea & - & $\mathrm{V}$ & Corylus avellana & III & - \\
\hline Carex riparia & I & $\mathrm{V}$ & Dactylis polygama & III & - \\
\hline Galium palustre & I & $\mathrm{V}$ & Elymus caninus & III & - \\
\hline Lycopus europaeus & II & $\mathrm{V}$ & Geranium robertianum & III & - \\
\hline Salix fragilis & II & $\mathrm{V}$ & Ophioglossum vulgatum & III & - \\
\hline Solanum dulcamara & II & $\mathrm{V}$ & Trollius europaeus & III & - \\
\hline Symphytum officinale & II & $\mathrm{V}$ & Veronica chamaedrys & III & - \\
\hline Carex acutiformis & III & $\mathrm{V}$ & Arctium minus & III & $\mathrm{I}$ \\
\hline Sub-constant species & & & Deschampsia caespitosa & III & I \\
\hline Cucubalus baccifer & IV & - & Populus tremula & III & $\mathrm{I}$ \\
\hline
\end{tabular}


Table 4 (continued)

\begin{tabular}{|c|c|c|c|c|c|}
\hline & $\mathrm{V}-\mathrm{Fr}$ & Fr-A & & V-Fr & Fr-A \\
\hline Sambucus nigra & III & I & Hypericum tetrapterum & II & - \\
\hline Bidens tripartita & - & III & Polygonatum latifolium & II & - \\
\hline Carex vesicaria & - & III & Pulmonaria mollissima & II & - \\
\hline Glyceria maxima & - & III & Ranunculus auricomus agg. & II & - \\
\hline Impatiens noli-tangere & - & III & Rosa canina agg. & II & - \\
\hline Lythrum salicaria & - & III & Rumex sanguineus & II & - \\
\hline Salix alba & - & III & Stachys sylvatica & II & - \\
\hline Sub-accessorial species & & & Vicia cracca & II & - \\
\hline Acer tataricum & II & - & Chenopodium polyspermum & - & II \\
\hline Alliaria petiolata & II & - & Myosotis nemorosa & - & II \\
\hline Angelica palustris & II & - & Persicaria hydropiper & - & II \\
\hline Campanula glomerata & II & - & Poa palustris & - & II \\
\hline Campanula trachelium & II & - & Scutellaria galericulata & - & II \\
\hline Carex michelii & II & - & Teucrium scordium & - & II \\
\hline Carex spicata & II & - & Thelypteris palustris & - & II \\
\hline Carex sylvatica & II & - & Typha latifolia & - & II \\
\hline Circaea lutetiana & II & - & Number of differential & 68 & 32 \\
\hline Cirsium rivulare & II & - & species & & \\
\hline Cruciata glabra & II & - & \multirow{4}{*}{\multicolumn{3}{|c|}{$\begin{array}{l}\text { V-Fr = Veratro albi-Fraxinetum angustifo- } \\
\text { liae (Kevey and Papp L. ined.: } 10 \text { rel.); } \\
\text { Fr-A = Fraxino pannonicae-Alnetum (Ke- } \\
\text { vey, Papp L. and Lendvai ined.: } 5 \text { rel.) }\end{array}$}} \\
\hline Fraxinus pennsylvanica & II & - & & & \\
\hline Galium mollugo & II & - & & & \\
\hline Heracleum sphondylium & II & - & & & \\
\hline
\end{tabular}

Fig. 10. Proportion of Molinio-Juncetea s. 1. character species. Fr-A = Fraxino pannonicae-Alnetum, Nyírség (Kevey and Papp L. ined.: 5 relevés); $\mathrm{V}-\mathrm{Fr}=$ Veratro albi-Fraxinetum angustifoliae, Nyírség (Kevey and Papp L. ined.: 10 relevés); Fr-U = Fraxino pannonicae-Ulmetum, Nyírség (Kevey et al. 2017: 20 relevés)

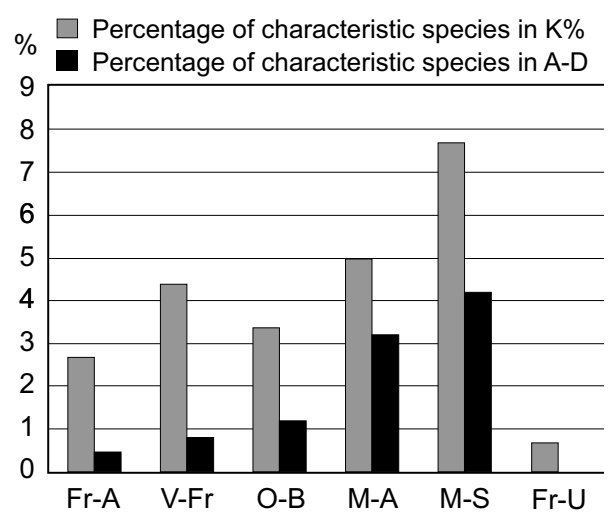


Table 5

Differential species in Veratro albi-Fraxinetum angustifoliae and Fraxino pannonicae-Ulmetum

\begin{tabular}{|c|c|c|c|c|c|}
\hline & V-Fr & Fr-U & & V-Fr & Fr-U \\
\hline Constant species & & & Taraxacum officinale agg. & IV & I \\
\hline Angelica sylvestris & $\mathrm{V}$ & - & Chelidonium majus & - & IV \\
\hline Equisetum arvense & $\mathrm{V}$ & - & Pulmonaria officinalis & - & IV \\
\hline Filipendula ulmaria & $\mathrm{V}$ & - & Carpinus betulus & $\mathrm{I}$ & IV \\
\hline Lychnis flos-cuculi & $\mathrm{V}$ & - & Lapsana communis & I & IV \\
\hline Eupatorium cannabinum & $\mathrm{V}$ & I & Ulmus laevis & I & IV \\
\hline Frangula alnus & $\mathrm{V}$ & $\mathrm{I}$ & \multirow{2}{*}{$\begin{array}{l}\text { Veronica hederifolia subsp. } \\
\text { lucorum }\end{array}$} & \multirow[t]{2}{*}{ I } & \multirow[t]{2}{*}{ IV } \\
\hline Veratrum album & $\mathrm{V}$ & I & & & \\
\hline Rhamnus catharticus & $\mathrm{V}$ & II & Viola mirabilis & I & IV \\
\hline Lysimachia nummularia & $\mathrm{V}$ & III & Alliaria petiolata & II & IV \\
\hline Populus alba & $\mathrm{V}$ & III & Carex sylvatica & II & IV \\
\hline Viburnum opulus & $\mathrm{V}$ & III & Festuca gigantea & II & IV \\
\hline Ranunculus ficaria & - & $\mathrm{V}$ & \multicolumn{3}{|l|}{ Accessorial species } \\
\hline Viola reichenbachiana & - & $\mathrm{V}$ & Betonica officinalis & III & - \\
\hline Acer campestre & I & $\mathrm{V}$ & Carex acutiformis & III & - \\
\hline Milium effusum & I & $\mathrm{V}$ & Cirsium canum & III & - \\
\hline Polygonatum multiflorum & I & $\mathrm{V}$ & Iris pseudacorus & III & - \\
\hline Ajuga reptans & II & $\mathrm{V}$ & Ophioglossum vulgatum & III & - \\
\hline Circaea lutetiana & II & $\mathrm{V}$ & Trollius europaeus & III & - \\
\hline Stachys sylvatica & II & $\mathrm{V}$ & Clinopodium vulgare & III & I \\
\hline Chaerophyllum temulum & III & $\mathrm{V}$ & Deschampsia caespitosa & III & I \\
\hline Convallaria majalis & III & $\mathrm{V}$ & Elymus caninus & III & I \\
\hline Corylus avellana & III & $\mathrm{V}$ & Populus tremula & III & I \\
\hline Geranium robertianum & III & $\mathrm{V}$ & Actaea spicata & - & III \\
\hline Sambucus nigra & III & $\mathrm{V}$ & Bromus ramosus agg. & - & III \\
\hline Sub-constant species & & & Fallopia dumetorum & - & III \\
\hline Geranium palustre & IV & - & Hedera helix & - & III \\
\hline Valeriana officinalis & IV & - & Lilium martagon & - & III \\
\hline Glechoma hederacea & IV & I & Tilia cordata & - & III \\
\hline Poa trivialis & IV & I & Tilia tomentosa & - & III \\
\hline Prunus spinosa & IV & I & Carex divulsa & I & III \\
\hline Pyrus pyraster & IV & I & Carex remota & I & III \\
\hline
\end{tabular}


Table 5 (continued)

\begin{tabular}{|c|c|c|c|c|c|}
\hline & $\mathrm{V}-\mathrm{Fr}$ & Fr-U & & $\mathrm{V}-\mathrm{Fr}$ & Fr-U \\
\hline Sub-accessorial species & & & Chaerophyllum aromaticum & - & II \\
\hline Angelica palustris & II & - & Colchicum autumnale & - & II \\
\hline Campanula glomerata & II & - & Corydalis cava & - & II \\
\hline Carex michelii & II & - & Dryopteris filix-mas & - & II \\
\hline Carex otrubae & II & - & Euphorbia amygdaloides & - & II \\
\hline Carex spicata & II & - & Galeobdolon luteum & - & II \\
\hline Cirsium rivulare & II & - & Galium odoratum & - & II \\
\hline Galium mollugo & II & - & Impatiens noli-tangere & - & II \\
\hline Hypericum tetrapterum & II & - & Lathraea squamaria & - & II \\
\hline Lycopus europaeus & II & - & Sanicula europaea & - & II \\
\hline Pulmonaria mollissima & II & - & Scilla vindobonensis & - & II \\
\hline Sium latifolium & II & - & Viola suavis s. 1. & - & II \\
\hline Symphytum officinale & II & - & Number of differential & 41 & 47 \\
\hline Vicia cracca & II & - & species & & \\
\hline Allium ursinum & - & II & \multirow{3}{*}{\multicolumn{3}{|c|}{$\begin{array}{l}\text { V-Fr = Veratro albi-Fraxinetum angustifoliae } \\
\text { (Kevey and Papp L. ined.: } 10 \text { rel.); Fr-U = } \\
\text { Fraxino pannonicae-Ulmetum, Nyírség } \\
\text { (Kevey and Papp L. ined.: } 20 \text { rel.) }\end{array}$}} \\
\hline Anemone ranunculoides & - & II & & & \\
\hline Cerasus avium & - & II & & & \\
\hline
\end{tabular}

Table 6

Differential species in Veratro albi-Fraxinetum angustifoliae and Ophioglosso-Betuletum

\begin{tabular}{|c|c|c|c|c|c|}
\hline & $\mathrm{V}-\mathrm{Fr}$ & O-B & & $\mathrm{V}-\mathrm{Fr}$ & $\mathrm{O}-\mathrm{B}$ \\
\hline \multicolumn{3}{|l|}{ Constant species } & Carex flacca & - & $\mathrm{V}$ \\
\hline Cornus sanguinea & $\mathrm{V}$ & - & Chelidonium majus & - & V \\
\hline Filipendula ulmaria & V & - & Cirsium oleraceum & - & V \\
\hline \multirow{2}{*}{$\begin{array}{l}\text { Fraxinus angustifolia } \\
\text { subsp. danubialis }\end{array}$} & \multirow[t]{2}{*}{$\mathrm{V}$} & \multirow[t]{2}{*}{-} & Clematis vitalba & - & V \\
\hline & & & Cynoglossum officinale & - & V \\
\hline Lychnis flos-cuculi & V & - & Dactylis glomerata & - & V \\
\hline Lysimachia nummularia & V & - & Fallopia dumetorum & - & V \\
\hline Scrophularia nodosa & $\mathrm{V}$ & - & Galium odoratum & - & $\mathrm{V}$ \\
\hline Populus alba & V & I & Molinia coerulea & - & V \\
\hline Equisetum arvense & V & II & Phragmites australis & - & V \\
\hline Galium aparine & V & II & Quercus cerris & - & V \\
\hline Acer pseudo-platanus & - & $\mathrm{V}$ & Ranunculus polyanthemos & - & $\mathrm{V}$ \\
\hline Arctium lappa & - & $\mathrm{V}$ & Sanguisorba officinalis & - & $\mathrm{V}$ \\
\hline
\end{tabular}


Table 6 (continued)

\begin{tabular}{|c|c|c|c|c|c|}
\hline & V-Fr & O-B & & V-Fr & O-B \\
\hline Solidago gigantea & - & V & Achillea millefolium & - & IV \\
\hline Acer campestre & I & V & Cardamine impatiens & - & IV \\
\hline Acer negundo & I & V & Cerasus avium & - & IV \\
\hline Betula pubescens & I & V & Festuca rubra & - & IV \\
\hline Galeopsis pubescens & I & V & Alliaria petiolata & II & IV \\
\hline Lapsana communis & I & V & Accessorial species & & \\
\hline Lysimachia vulgaris & I & V & Arctium minus & III & - \\
\hline Mycelis muralis & I & V & Betonica officinalis & III & - \\
\hline Ranunculus acris & I & V & Cirsium canum & III & - \\
\hline Selinum carvifolia & I & V & Clinopodium vulgare & III & - \\
\hline Serratula tinctoria & I & V & Convallaria majalis & III & - \\
\hline Circaea lutetiana & II & V & Corylus avellana & III & - \\
\hline Festuca gigantea & II & V & Dactylis polygama & III & - \\
\hline Fraxinus pennsylvanica & II & V & Elymus caninus & III & - \\
\hline Galium mollugo & II & V & Iris pseudacorus & III & - \\
\hline Humulus lupulus & II & V & Populus tremula & III & - \\
\hline Rosa canina agg. & II & V & Trollius europaeus & III & - \\
\hline Stachys sylvatica & II & V & Veronica chamaedrys & III & - \\
\hline Carex acutiformis & III & V & Agrimonia eupatoria & - & III \\
\hline Chaerophyllum temulum & III & V & Colchicum autumnale & - & III \\
\hline Deschampsia caespitosa & III & V & Dianthus superbus & - & III \\
\hline Geranium robertianum & III & V & Mentha pulegium & - & III \\
\hline Ophioglossum vulgatum & III & V & Poa angustifolia & - & III \\
\hline Sambucus nigra & III & V & Rubus fruticosus agg. & - & III \\
\hline Urtica dioica & III & V & Tussilago farfara & - & III \\
\hline Sub-constant species & & & Lathyrus pratensis & $\mathrm{I}$ & III \\
\hline Geranium palustre & IV & - & Sub-accessorial species & & \\
\hline Glechoma hederacea & IV & - & Acer tataricum & II & - \\
\hline Listera ovata & IV & - & Ajuga reptans & II & - \\
\hline Ulmus minor & IV & - & Angelica palustris & II & - \\
\hline Valeriana officinalis & IV & - & Caltha palustris & II & - \\
\hline Poa trivialis & IV & I & Campanula glomerata & II & - \\
\hline Quercus robur & IV & I & Campanula trachelium & II & - \\
\hline Prunus spinosa & IV & II & Cardamine pratensis & II & - \\
\hline
\end{tabular}


Table 6 (continued)

\begin{tabular}{|c|c|c|c|c|c|}
\hline & $\mathrm{V}-\mathrm{Fr}$ & $\mathrm{O}-\mathrm{B}$ & & V-Fr & $\mathrm{O}-\mathrm{B}$ \\
\hline Carex michelii & II & - & Sium latifolium & II & - \\
\hline Carex otrubae & II & - & Symphytum officinale & II & - \\
\hline Carex spicata & II & - & Vicia cracca & II & - \\
\hline Carex sylvatica & II & - & Agrostis stolonifera & - & II \\
\hline Cirsium rivulare & II & - & Bromus ramosus agg. & - & II \\
\hline Cruciata glabra & II & - & Euphorbia cyparissias & - & II \\
\hline Heracleum sphondylium & II & - & Phleum pratense & - & II \\
\hline Hypericum tetrapterum & II & - & Valeriana dioica & - & II \\
\hline Lycopus europaeus & II & - & Viola reichenbachiana & - & II \\
\hline Pulmonaria mollissima & II & - & Number of differential & 52 & 59 \\
\hline Ranunculus auricomus agg. & II & - & & & נע \\
\hline $\begin{array}{l}\text { Rumex sanguineus } \\
\text { Salix fragilis }\end{array}$ & $\begin{array}{l}\text { II } \\
\text { II }\end{array}$ & - & \multicolumn{3}{|c|}{$\begin{array}{l}\mathrm{V}-\mathrm{Fr}=\text { Veratro albi-Fraxinetum angustifo- } \\
\text { liae (Kevey and Papp L. ined.: } 10 \text { rel.); } \\
\mathrm{O}-\mathrm{B}=\text { Ophioglosso-Betuletum }\end{array}$} \\
\hline
\end{tabular}

Fraxino pannonicae-Ulmetum, which reflects the transitional habitat of Veratro albi-Fraxinetum angustifoliae between the former two (Table 3, Figs 5-10). The two exceptions are the Quercetea pubescentis-petraeae s. 1. and Molinio-Juncetea s. l. species. The proportion of the former is the highest not only among the three, but among all six communities, whereas that of the latter is the highest among the three hardwood riparian forest associations, but is the smallest among all drained swamp communities. The distribution of character species proportions in Veratro albi-Fraxinetum pannonicae are rather different from the latter communities. The largest differences are found in the character species of Phragmitetea s. 1., Molinio-Juncetea s. 1., Galio-Urticetea s. 1., Epilobietea s. 1., Salicetea purpureae s. 1., Alnetea glutinosae s. 1., Querco-Fagetea s. 1., Fagetalia, Alnion incanae s. 1. and Quercetea pubescentis-petraeae s. 1. (Table 4).

The occurrence of introduced aliens (Fraxinus pennsylvanica, Acer negundo, Celtis occidentalis, Echinocystis lobata, Parthenocissus inserta, Robinia pseudoacacia, Vitis riparia) in the association is apparent (Table 1), but compared to other associations in the region, their proportion is low (1.9\%).

\section{Number of differentiating species}

The number of differentiating species (species for which the difference between their constancy values in the compared two communities equals or exceeds two) in Veratro albi-Fraxinetum angustifoliae is greater than 40 in all pairwise comparisons. The highest number of differentiating species (68) was 
Table 7

Differential species in Veratro albi-Fraxinetum angustifoliae and Molinio-Alnetum glutinosae

\begin{tabular}{|c|c|c|c|c|c|}
\hline & $\mathrm{V}-\mathrm{Fr}$ & M-A & & V-Fr & M-A \\
\hline \multicolumn{3}{|l|}{ Constant species } & \multicolumn{3}{|l|}{ Sub-constant species } \\
\hline Filipendula ulmaria & $\mathrm{V}$ & - & Geranium palustre & IV & - \\
\hline \multirow{2}{*}{$\begin{array}{l}\text { Fraxinus angustifolia } \\
\text { subsp. danubialis }\end{array}$} & \multirow[t]{2}{*}{$\mathrm{V}$} & \multirow[t]{2}{*}{-} & Listera ovata & IV & - \\
\hline & & & Moehringia trinervia & IV & - \\
\hline \multirow{2}{*}{$\begin{array}{l}\text { Scrophularia nodosa } \\
\text { Lychnis flos-cuculi }\end{array}$} & $\begin{array}{l}\mathrm{V} \\
\mathrm{V}\end{array}$ & - & Glechoma hederacea & IV & I \\
\hline & $\mathrm{V}$ & I & Ulmus minor & IV & II \\
\hline Torilis japonica & $\mathrm{V}$ & I & Pyrus pyraster & IV & III \\
\hline Veratrum album & $\mathrm{V}$ & I & Asclepias syriaca & - & IV \\
\hline Euonymus europaeus & $\mathrm{V}$ & II & Carex flacca & - & IV \\
\hline Populus alba & $\mathrm{V}$ & II & Cynoglossum hungaricum & - & IV \\
\hline Geum urbanum & $\mathrm{V}$ & III & Equisetum palustre & - & IV \\
\hline Ligustrum vulgare & $\mathrm{V}$ & III & Lythrum salicaria & - & IV \\
\hline Lysimachia nummularia & $\mathrm{V}$ & III & Phragmites australis & - & IV \\
\hline Viburnum opulus & $\mathrm{V}$ & III & Potentilla reptans & - & IV \\
\hline Alnus glutinosa & - & $\mathrm{V}$ & Ranunculus polyanthemos & - & IV \\
\hline Molinia coerulea & - & $\mathrm{V}$ & Sonchus palustris & - & IV \\
\hline Poa pratensis & - & $\mathrm{V}$ & Galium palustre & I & IV \\
\hline Salix cinerea & - & $\mathrm{V}$ & Mentha aquatica & I & IV \\
\hline Sanguisorba officinalis & - & $\mathrm{V}$ & Selinum carvifolia & I & IV \\
\hline Solidago gigantea & - & $\mathrm{V}$ & Stachys palustris & $\mathrm{I}$ & IV \\
\hline Valeriana dioica & - & $\mathrm{V}$ & Caltha palustris & II & IV \\
\hline Calystegia sepium & I & $\mathrm{V}$ & Rosa canina agg. & II & IV \\
\hline Celtis occidentalis & I & $\mathrm{V}$ & Accessorial species & & \\
\hline Lysimachia vulgaris & I & $\mathrm{V}$ & Clinopodium vulgare & III & - \\
\hline Ranunculus acris & I & $\mathrm{V}$ & Convallaria majalis & III & - \\
\hline Lycopus europaeus & II & $\mathrm{V}$ & Corylus avellana & III & - \\
\hline Ranunculus repens & II & $\mathrm{V}$ & Trollius europaeus & III & - \\
\hline Solanum dulcamara & II & $\mathrm{V}$ & Betonica officinalis & III & $\mathrm{I}$ \\
\hline Vicia cracca & II & V & Dactylis polygama & III & I \\
\hline Carex acutiformis & III & $\mathrm{V}$ & Elymus caninus & III & I \\
\hline Cirsium canum & III & $\mathrm{V}$ & Ophioglossum vulgatum & III & I \\
\hline Deschampsia caespitosa & III & $\mathrm{V}$ & Veronica chamaedrys & III & I \\
\hline Iris pseudacorus & III & $\mathrm{V}$ & Carex elata & - & III \\
\hline Sambucus nigra & III & $\mathrm{V}$ & & & \\
\hline
\end{tabular}


Table 7 (continued)

\begin{tabular}{|c|c|c|c|c|c|}
\hline & V-Fr & M-A & & $\mathrm{V}-\mathrm{Fr}$ & M-A \\
\hline Galium boreale & - & III & Allium angulosum & - & II \\
\hline Galium verum & - & III & Bromus sterilis & - & II \\
\hline Genista tinctoria subsp. & - & III & Carex paniculata & - & II \\
\hline & & & Cirsium arvense & - & II \\
\hline Phalaris arundinacea & - & III & Cirsium vulgare & - & II \\
\hline Euphorbia palustris & I & III & Dactylis glomerata & - & II \\
\hline Succisa pratensis & I & III & Festuca pratensis & - & II \\
\hline Sub-accessorial species & & & Gentiana pneumonanthe & - & II \\
\hline Acer tataricum & II & - & Iris sibirica & - & II \\
\hline Ajuga reptans & II & - & Leucojum aestivum & - & II \\
\hline Angelica palustris & II & - & Morus alba & - & II \\
\hline Campanula glomerata & II & - & Padus serotina & - & II \\
\hline Campanula trachelium & II & - & Phytolacca americana & - & II \\
\hline Cardamine pratensis & II & - & Pulicaria dysenterica & - & II \\
\hline Carex otrubae & II & - & Salix alba & - & II \\
\hline Carex spicata & II & - & Salix rosmarinifolia & - & II \\
\hline Carex sylvatica & II & - & Scirpoides holoschoenus & - & II \\
\hline Cirsium rivulare & II & - & Scutellaria galericulata & - & II \\
\hline Cruciata glabra & II & - & Thalictrum flavum & - & II \\
\hline Heracleum sphondylium & II & - & Trifolium montanum & - & II \\
\hline Pulmonaria mollissima & II & - & Number of differential & 43 & 63 \\
\hline Ranunculus auricomus agg. & II & - & species & 43 & 63 \\
\hline Rumex sanguineus & II & - & \multirow{3}{*}{\multicolumn{3}{|c|}{$\begin{array}{l}\text { V-Fr }=\text { Veratro albi-Fraxinetum angus- } \\
\text { tifoliae (Kevey and Papp L. ined.: } 10 \\
\text { rel.); M-A = Molinio-Alnetum glutinosae, } \\
\text { Mezőföld (Kevey 2008: } 20 \text { rel.) }\end{array}$}} \\
\hline Stachys sylvatica & II & - & & & \\
\hline Agrimonia eupatoria & - & II & & & \\
\hline
\end{tabular}

found in the comparison to the alder swamp (Fraxino-Alnetum glutinosae), whereas the smallest number (41) occurred in relation to Fraxino pannonicaeUlmetum (Tables 5-8).

\section{Number of protected species}

The stands of Veratro albi-Fraxinetum angustifoliae host numerous rare, threatened, or otherwise protected plant species. These are: Veratrum album (K: V), Listera ovata (K: IV) Ophioglossum vulgatum, Trollius europaeus (both 
K: III), Betula pubescens, Cephalanthera damasonium, C. longifolia, Epipactis helleborine agg., Equisetum hyemale (incl. Equisetum $\times$ moorei), Orchis militaris, Platanthera bifolia (all K: I).

\section{Similarity relations in multivariate analyses}

In the cluster analysis with complete linkage algorithm, the ten samples of Veratro albi-Fraxinetum angustifoliae grouped with the samples of Fraxino pannonicae-Ulmetum. The rest of the samples formed the sister cluster of this group. In this cluster, samples of the alder swamp (Fraxino pannonicae-Alnetum) were the sister cluster of the three drained swamp communities (OphioglossoBetuletum pubescentis, Molinio-Alnetum, Molinio-Salicetum cinereae) (Fig. 11). The result in the analysis with the group-average algorithm differed only in the placement of one of the drained swamp communities (Ophioglosso-Betuletum

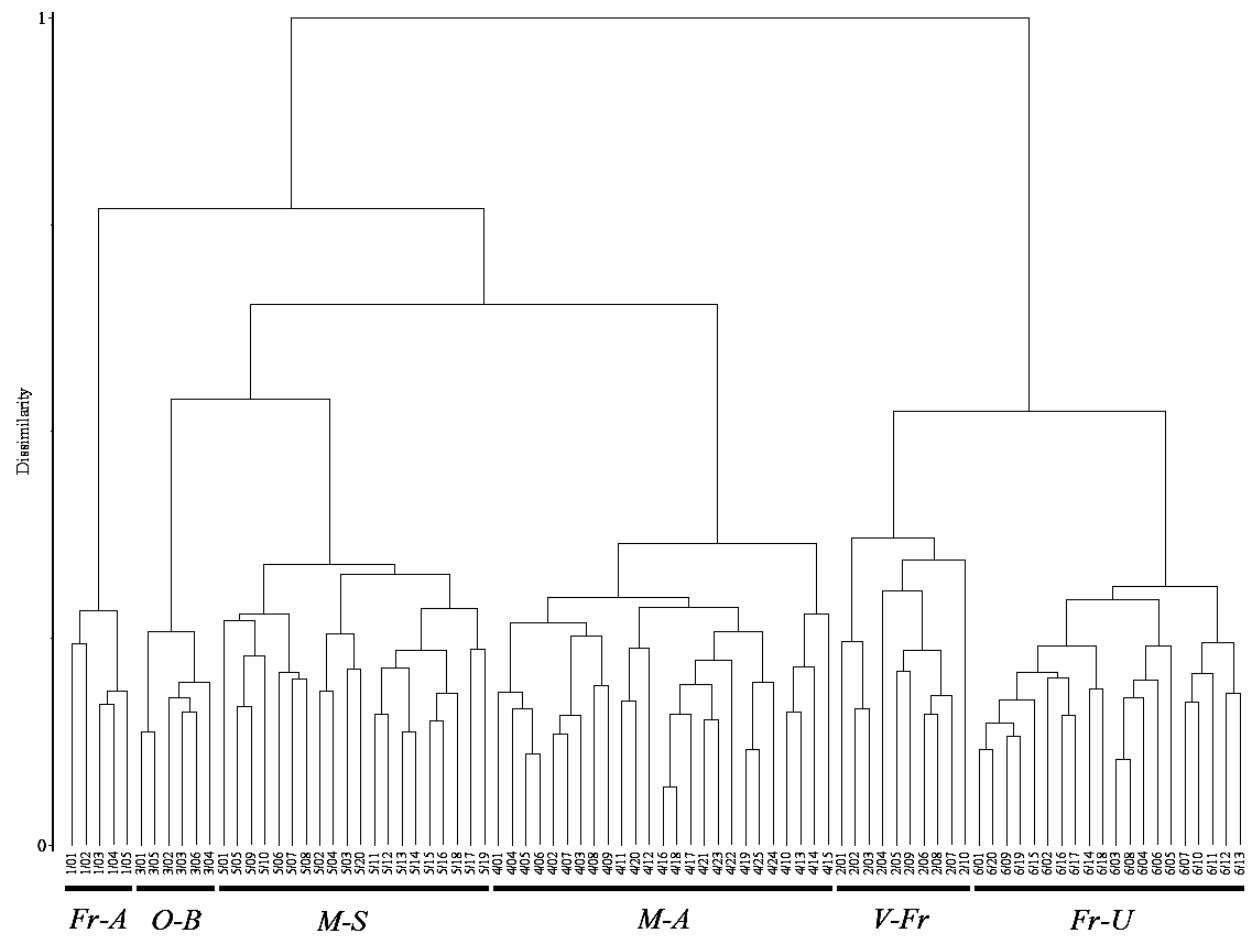

Fig. 11. Binary dendrogram of alder swamp, hardwood riparian forest and drained swamp communities I (method: complete link; coefficient: Baroni-Urbani and Buser). $1 / 1-5=$ Fraxino pannonicae-Alnetum, Nyírség (Kevey and Papp L. ined.); 2/1-10 = Veratro albi-Fraxinetum angustifoliae, Nyírség (Kevey and Papp L. ined.);3/1-6 = Ophioglosso-Betuletum pubescentis, Vértesalja (Riezing and Szollát 2008-2009); 4/1-25 = Molinio-Salicetum cinereae, Szigetköz (Kevey 2008); 5/1-20 = Molinio-Alnetum glutinosae, Mezőföld (Kevey 2008); 6/1-20 = Fraxino pannonicae-Ulmetum, Nyírség (Kevey et al. 2017) 
pubescentis) as a sister group of the Veratro albi-Fraxinetum and Fraxino pannonicae-Ulmetum cluster (Fig. 12). The dissimilarity level of the Veratro albi-Fraxinetum and Fraxino pannonicae-Ulmetum was very similar, though slightly smaller than those between the Ophioglosso-Betuletum and Molinio-Alnetum (complete linkage), and Molinio-Alnetum and Molinio-Salicetum cinereae (group average).

The result of the PCoA is in agreement with the above. In the plane of axes one and two, the samples of Veratro albi-Fraxinetum were adjacent to both, Fraxino pannonicae-Ulmetum and Ophioglosso-Betuletum pubescentis (Fig. 13). However, the position of the latter changed substantially in the plane of axes one and three, while the spatial relation of the samples of Veratro albi-Fraxinetum and Fraxino pannonicae-Ulmetum essentially did not change (Fig. 14).

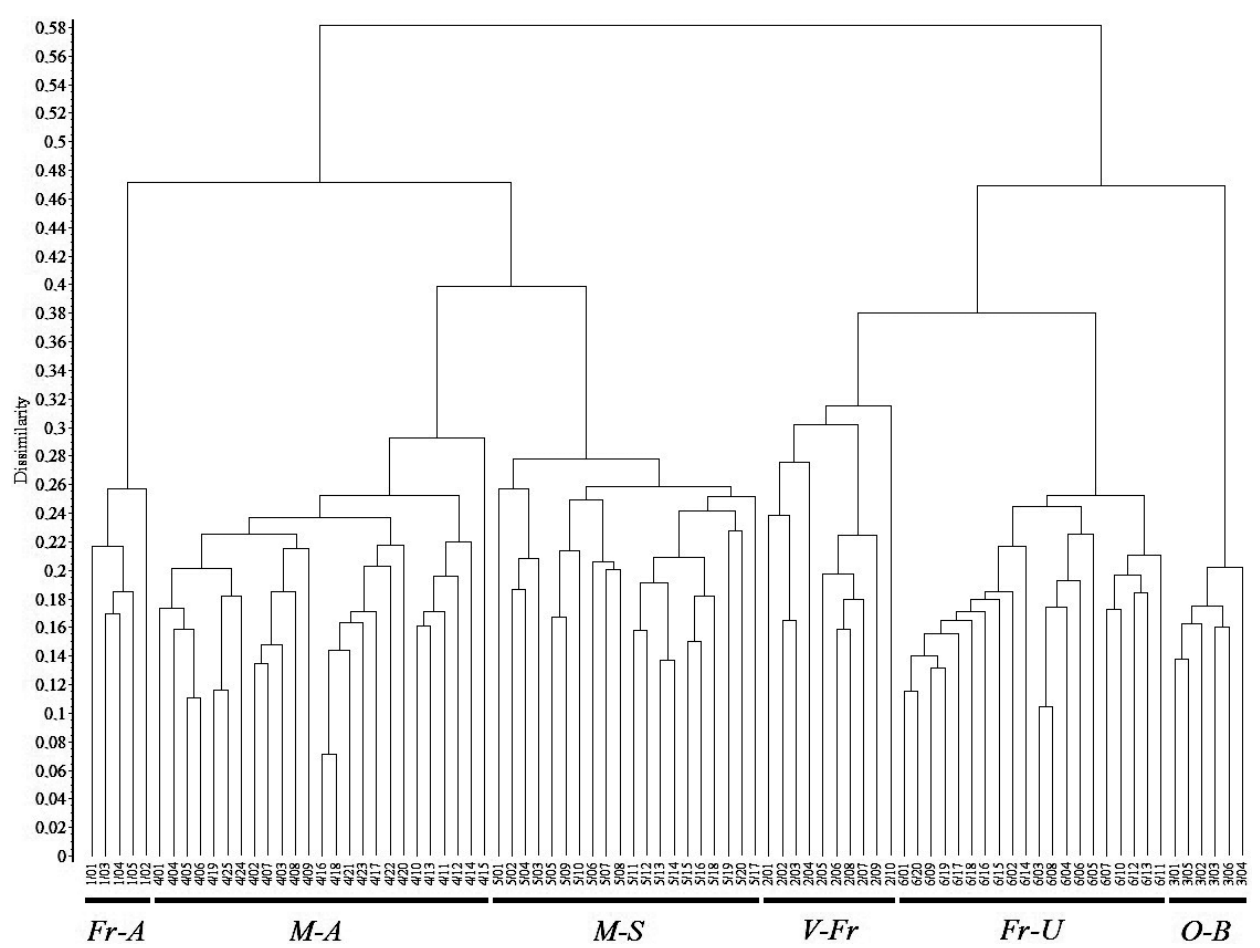

Fig. 12. Binary dendrogram of alder swamp, hardwood riparian forest and drained swamp communities II (method: group average; coefficient: Baroni-Urbani and Buser). 1/1-5: Fraxino pannonicae-Alnetum, Nyírség (Kevey and Papp L. ined.); 2/1-10: Veratro albi-Fraxinetum angustifoliae, Nyírség (Kevey and Papp L. ined.); 3/1-6: Ophioglosso-Betuletum pubescentis, Vértesalja (Riezing and Szollát 2008-2009); 4/1-25: Molinio-Salicetum cinereae, Szigetköz (Kevey 2008); 5/1-20: Molinio-Alnetum glutinosae, Mezőföld (Kevey 2008); 6/1-20: Fraxino pannonicae-Ulmetum, Nyírség (Kevey et al. 2017) 


\section{DISCUSSION}

Field observations on habitat characteristics of Veratro albi-Fraxinetum angustifoliae suggested its transitional nature between Fraxino pannonicae-Alnetum and Fraxino pannonicae-Ulmetum. It is likely that this community develops from Fraxino pannonicae-Alnetum as organic and inorganic deposits accumulate and the habitat gradually dries out. During this process, most species of the Lemno-Potametea s. 1. class, and also many Phragmitetea s. 1. and Alnetea glutinosae s. 1. species disappear or are replaced by Molinietalia s. 1., QuercoFagetea and Quercetea pubescenti-petraeae species. This genealogic relationship would manifest itself in similarities in species composition and character species proportions to both associations.

It also seemed reasonable to assume that this association may be closely related to drained swamp communities based on their similarities in habitat conditions. The habitat of these communities may be covered with water in wet periods, but the soil is typically not saturated with water most of the time. This allows the establishment of Molinietalia and Quercetea species in relatively high proportions. These communities also share a number of additional

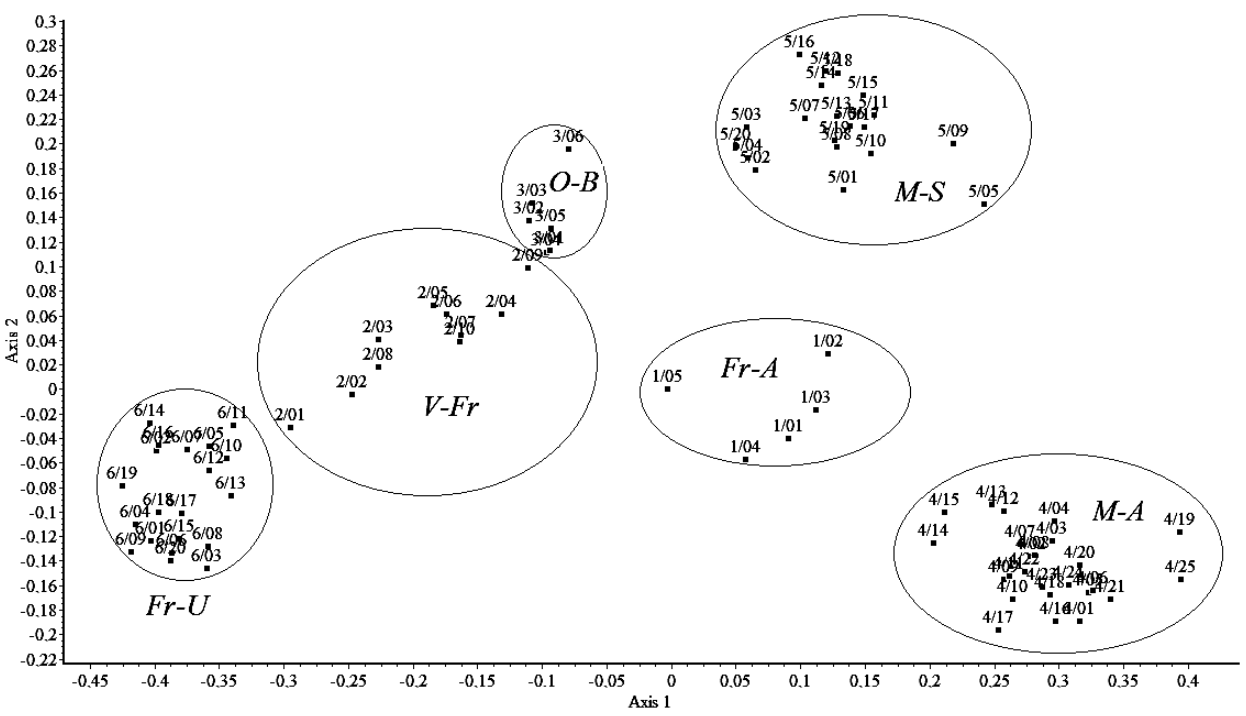

Fig. 13. Ordination diagram (axes 1 and 2) of alder swamp, hardwood riparian forest and drained swamp communities I (method: principal coordinates analysis; coefficient: BaroniUrbani and Buser). 1/1-5: Fraxino pannonicae-Alnetum, Nyírség (Kevey and Papp L. ined.); 2/1-10: Veratro albi-Fraxinetum angustifoliae, Nyírség (Kevey and Papp L. ined.);3/1-6: Ophioglosso-Betuletum pubescentis, Vértesalja (Riezing and Szollát 2008-2009); 4/1-25: MolinioSalicetum cinereae, Szigetköz (Kevey 2008); 5/1-20: Molinio-Alnetum glutinosae, Mezőföld (Kevey 2008); 6/1-20: Fraxino pannonicae-Ulmetum, Nyírség (Kevey et al. 2017) 
features including the relatively high proportions of Phragmitetea and Alnetalia glutinosae, and the low proportion of Fagetalia elements.

Despite these similarities, the Veratro albi-Fraxinetum angustifoliae cannot be identified with any of the studied associations, but is best recognised as a novel association. Its distinctiveness is supported by a suite of evidence, including the number of differentiating species, the distribution of character species proportions, and the dissimilarity in floristical composition. In our

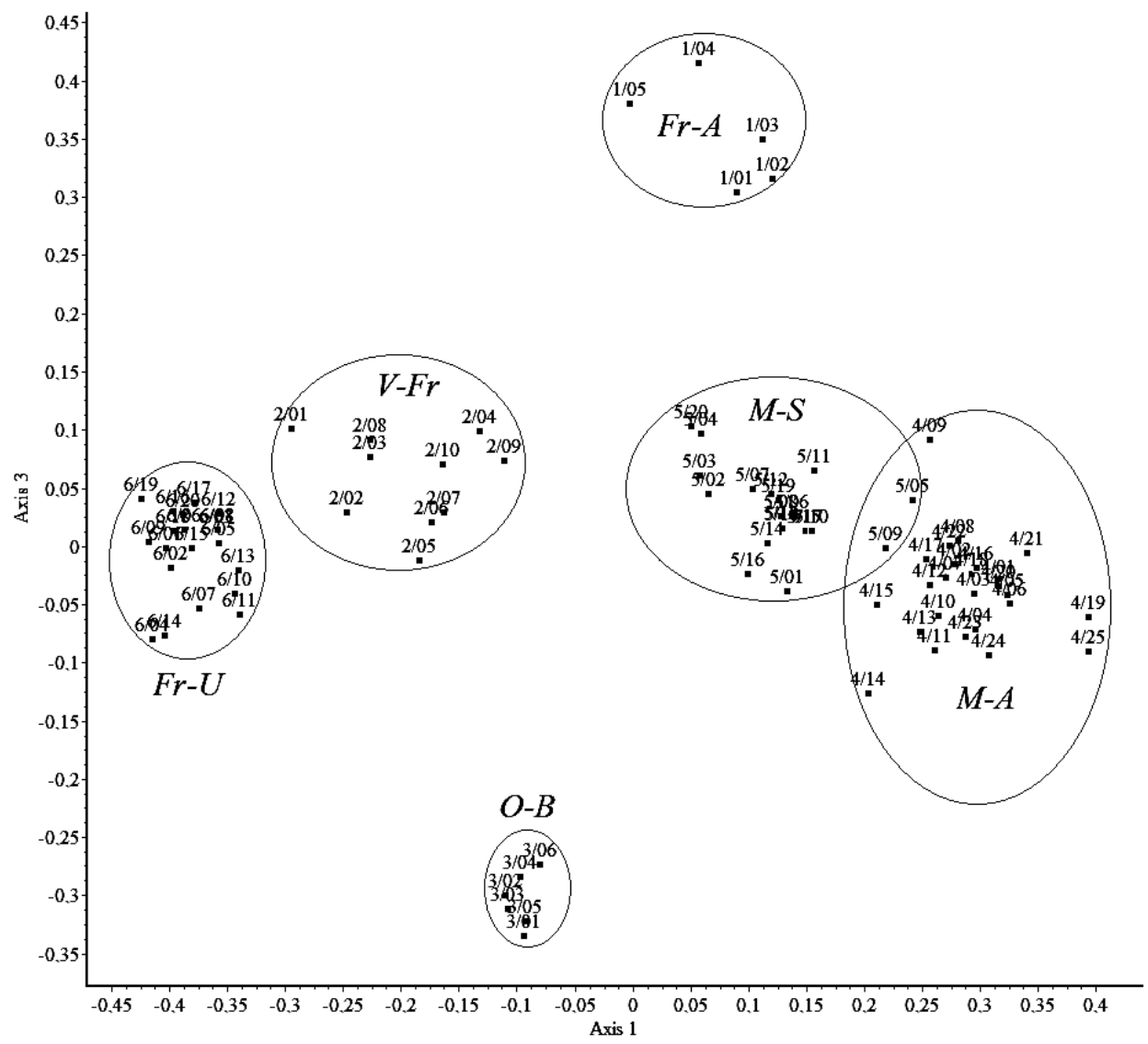

Fig. 14. Ordination diagram (axes 1 and 3) of alder swamp, hardwood riparian forest and drained swamp communities II (method: principal coordinates analysis; coefficient: Baroni-Urbani and Buser). 1/1-5: Fraxino pannonicae-Alnetum, Nyírség (Kevey and Papp L. ined.); 2/1-10: Veratro albi-Fraxinetum angustifoliae, Nyírség (Kevey and Papp L. ined.); 3/1-6: Ophioglosso-Betuletum pubescentis, Vértesalja (Riezing and Szollát 2008-2009); 4/125: Molinio-Salicetum cinereae, Szigetköz (Kevey 2008); 5/1-20: Molinio-Alnetum glutinosae, Mezőföld (Kevey 2008); 6/1-20: Fraxino pannonicae-Ulmetum, Nyírség (Kevey et al. 2017) 
opinion, the amount of differences in these features are sufficiently high to designate this community as a distinct association.

Whereas the Veratro albi-Fraxinetum angustifoliae is best treated as a new association, its syntaxonomic affinity is rather difficult to ascertain. Owing to its intermediate characteristics in many respects, it could be placed either in the Alnetea or the Querco-Fagetea class. The Molinio-Betuletea class by Passarge and Hofmann (1968), which includes strongly acidophilic communities distributed over the more humid western and northern parts of Europe, may be excluded for this reason. Based on preliminary data, Kevey (2008) placed the Veratro albi-Fraxinetum angustifoliae in the class Alnetea glutinosae, within an alliance Molinio-Alnion glutinosae, established for basiphilic drained swamp communities.

Abbreviations: $\mathrm{A} 1=$ upper forest canopy layer; $\mathrm{A} 2=$ lower forest canopy layer; $\mathrm{A} i=$ Alnion incanae; $A$ on $=$ Alnion glutinosae; $\mathrm{AQ}=$ Aceri tatariciQuercion; $\mathrm{Ar}=$ Artemisietea; $\mathrm{Ara}=$ Arrhenatheretea; $\mathrm{Arn}=$ Arrhenatherion elatioris; Ate $=$ Alnetea glutinosae; $\mathrm{B} 1=$ shrub layer; $\mathrm{B} 2$ = saplings; $\mathrm{Bec}=\mathrm{Beck}-$ mannion eruciformis; Ber $=$ Berberidion; Bia $=$ Bidentetea; Bin $=$ Bidention tripartiti; $\mathrm{C}=$ herbaceous layer; $\mathrm{Cal}=$ Calystegion sepium; $\mathrm{Cgr}=$ Caricenion gracilis; $\mathrm{ChS}=$ Chenopodio-Scleranthea; $\mathrm{Cp}=$ Carpinenion betuli; $\mathrm{Des}=\mathrm{De}-$ schampsion caespitosae; Epa = Epilobietea angustifolii; Epn $=$ Epilobion angustifolii; F = Fagetalia sylvaticae; FBt = Festuco-Brometea; $\mathrm{FiC}=$ FilipenduloCirsion oleracei; $\mathrm{FPi}=$ Festuco-Puccinellietalia; $\mathrm{Fr}-\mathrm{A}=$ alder swamp (Fraxino pannonicae-Alnetum); Fr-U = hardwood riparian forest (Fraxino pannonicae-Ulmetum); Fvg = Festucetea vaginatae; Fvl = Festucetalia valesiacae; GA = GalioAlliarion; incl. = inclusive; ined. $=$ ineditum (unpublished); $\mathrm{M}-\mathrm{A}=$ drained alder swamp (Molinio-Alnetum glutinosae); M-S = drained willow swamp (Molinio-Salicetum cinereae); Mag = Magnocaricetalia; Moa = Molinietalia coeruleae; $\mathrm{MoA}=$ Molinio-Arrhenatherea; MoJ = Molinio-Juncetea; Mon = Molinion coeruleae; $\mathrm{NC}=$ Nardo-Callunetea; $\mathrm{NG}=$ Nasturtio-Glycerietalia; $\mathrm{O}-\mathrm{B}=$ drained birch swamp (Ophioglosso-Betuletum pubescentis); Pla = Plantaginetea; Pna $=$ Populenion nigro-albae; $P Q=$ Pino-Quercetalia; $P r f=$ Prunion fruticosae; Pru $=$ Prunetalia spinosae; Pte $=$ Phragmitetea; $Q c=$ Quercetalia cerridis; $\mathrm{QFt}=\mathrm{Querco}-$ Fagetea; $\mathrm{Qpp}=$ Quercetea pubescentis-petraeae; $\mathrm{Qr}=$ Quercetalia roboris; Qrp = Quercion robori-petraeae; $\mathrm{S}=$ summa (sum); Sal = Salicion albae; SCn = Scheuchzerio-Caricetea nigrae; Sea = Secalietea; s. 1. = sensu lato (in the broad sense); Spu = Salicetea purpureae; s. str. = sensu stricto (in the narrow sense); Tof = Tofieldietalia; Ulm = Ulmenion; US = Urtico-Sambucetea, $\mathrm{V}-\mathrm{Fr}=$ drained ash swamp (Veratro albi-Fraxinetum angustifoliae). 
Acknowledgements - We thank László Papp junior and Endre Papp for their help during our fieldwork, and the two anonymous reviewers for their help improving the manuscript.

\section{REFERENCES}

Aszód, L. (1935): Adatok a nyírségi homoki vegetáció ökológiájához és szociológiájához. - Tisia 1(1): 1-33.

Becking, R. W. (1957): The Zürich-Montpellier School of phytosociology. - Bot. Review 23: 411-488.

Borhidi, A. (1961): Klimadiagramme und klimazonale Karte Ungarns. - Ann. Univ. Sci. Budapest., Sect. Biol. 4: 21-250.

Borhidi, A. (1993): A magyar flóra szociális magatartás típusai, természetességi és relatív ökológiai értékszámai. - Janus Pannonius Tudományegyetem, Pécs, 95 pp.

Borhidi, A. (1995): Social behaviour types, the naturalness and relative ecological indicator values of the higher plants in the Hungarian flora. - Acta Bot. Acad. Sci. Hung. 39: 97-181.

Borhidi, A. and Kevey, B. (1996): An annotated checklist of the Hungarian plant communities II. - In: Borhidi, A. (ed.): Critical revision of the Hungarian plant communities. Janus Pannonius University, Pécs, pp. 95-138.

Borhidi, A., Kevey, B. and Lendvai, G. (2012): Plant communities of Hungary. - Akadémiai Kiadó, Budapest, 544 pp.

Braun-Blanquet, J. (1964): Pflanzensoziologie. (ed. 3). - Springer Verlag, Wien, New York, $865 \mathrm{pp}$.

Du Rietz, G. E. (1921): Zur methodologischen Grundlage der modernen Pflanzensoziologie. Akademisk Afhandling, Uppsala, 272 pp.

Ellenberg, H. (1986): Vegetation Mitteleuropas mit den Alpen in ökologischer Sicht. (ed. 4). - Eugen Ulmer Verlag, Stuttgart, 989 pp.

Horvat, I. (1938): Biljnosociološka istraživanja šuma u Hrvatskoj. - Annales pro experimentis foresticis Zagreb 6: 127-279.

Horvat, I., Glavač, V. and Ellenberg, H. (1974): Vegetation Südosteuropas. - Gustav Fischer Verlag, Stuttgart, $768 \mathrm{pp}$.

Horváth, F., Dobolyi, Z. K., Morschhauser, T., Lőkös, L., Karas, L. and Szerdahelyi, T. (1995): Flóra adatbázis 1.2. - Vácrátót, 267 pp.

Járai-Komlódi, M. (1958): Die Pflanzengesellschaften in dem Turjángebiet von Ócsa-Dabas. - Acta Bot. Acad. Sci. Hung. 4: 63-92.

Kevey, B. (2008): Magyarország erdőtársulásai. (Forest associations of Hungary). - Tilia 14: $1-488$.

Kevey, B. and Hirmann, A. (2002): „NS” számítógépes cönológiai programcsomag. - In: Horváth, A. (ed.): Összefoglalók. Aktuális flóra- és vegetációkutatások a Kárpát-medencében V, Pécs, 2002. március 8-10., p. 74.

Kevey, B., Papp, L. and Lendvai, G. (2017): A Nyírség tölgy-kőris-szil ligetei (Fraxino pannonicae-Ulmetum Soó in Aszód 1935 corr. Soó 1963). - Kitaibelia 22(1): 179-220. https://doi.org/10.17542/kit.22.179

Király, G. (ed.) (2009): Új magyar füvészkönyv. Magyarország hajtásos növényei. Határozókulcsok. - Aggteleki Nemzeti Park Igazgatóság, Jósvafő, 616 pp. 
Mucina, L., Grabherr, G. and Wallnöfer, S. (1993): Die Pflanzengesellschaften Österreichs III. Wälder und Gebüsche. - Gustav Fischer, Jena, Stuttgart, New York, 353 pp.

Oberdorfer, E. (1992a): Süddeutsche Pflanzengesellschaften IV. A. Textband. - Gustav Fischer Verlag, Jena, Stuttgart, New York, 282 pp.

Oberdorfer, E. (1992b): Süddeutsche Pflanzengesellschaften IV. B. Tabellenband. - Gustav Fischer Verlag, Jena, Stuttgart, New York, 580 pp.

Papp, L. and Dudás, M. (1992): Data on botanical values of central and south Nyírség and their vicinity. - Déri Múzeum Évkönyve, Debrecen, 1989-1990: 7-35.

Passarge, H. and Hofmann, G. (1968): Molinio-Betuletea pubescentis. - In: Passarge, H. and Hofmann, G. (eds): Pflanzengesellschaften des nordostdeutschen Flachlandes II. VEB Gustav Fischer Verlag, Jena, pp. 190-199.

Podani J. (2001): Syntax 2000, computer programs for data analysis in ecology and systematics. Scientia, Budapest, $53 \mathrm{pp}$.

Riezing, N. and Szollát, Gy. (2008-2009): Kiszáradó nyírlápok a Vértesalján (OphioglossoBetuletum pubescentis Riezing, Szollát and Simon ass. nova). - Kanitzia 16(3-4): 45-58.

Rodwell, J. S., Schaminée, J. H. J., Mucina, L., Pignatti, S., Dring, J. and Moss, D. (2002): The diversity of European vegetation. - Wageningen, Wateringen, 168 pp.

Soó, R. (1964, 1966, 1968, 1970, 1973, 1980): A magyar flóra és vegetáció rendszertani-növényföldrajzi kézikönyve I-VI. - Akadémiai Kiadó, Budapest.

Tüxen, R. (1937): Die Pflanzengesellschaften Nordwestdeutschlands. - Mitt. Flor.-Soziol. Arbeitsgem. Niedersachsen, Hannover 3: 1-170.

Westhoff, V., Dijk, J. W. and Passchier, H. (1946): Overzicht der plantengemeenschappen in Nederland. - Tweede druk, G. W. Breughel, Amsterdam, 118 pp.

Willner, W. and Grabherr, G. (2007a): Die Wälder und Gebüsche Österreichs 1 Textband. - Elsevier Spektrum Akademischer Verlag, München, 302 pp.

Willner, W. and Grabherr, G. (2007b): Die Wälder und Gebüsche Österreichs. Tabellenband. Elsevier Spektrum Akademischer Verlag, München, 290 pp.

Open Acces statement. This is an open-access article distributed under the terms of the Creative Commons Attribution 4.0 International License (https://creativecommons.org/ licenses/by/4.0/), which permits unrestricted use, distribution, and reproduction in any medium, provided the original author and source are credited, a link to the CC License is provided, and changes - if any - are indicated. (SID_1) 

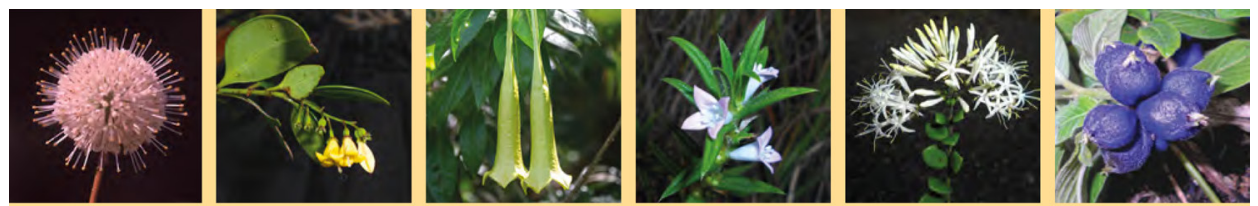

Attila Borhidi

Maira Fernández-Zequeira

Ramona Oviedo-Prieto

\section{Rubiáceas de Cuba}
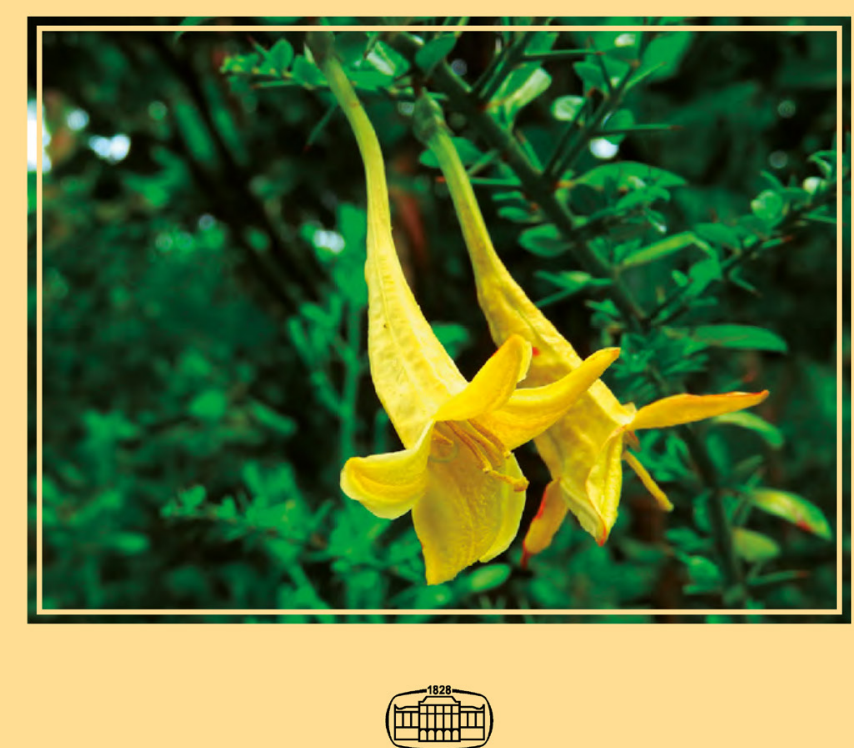

AKADÉMIAI KIADÓ

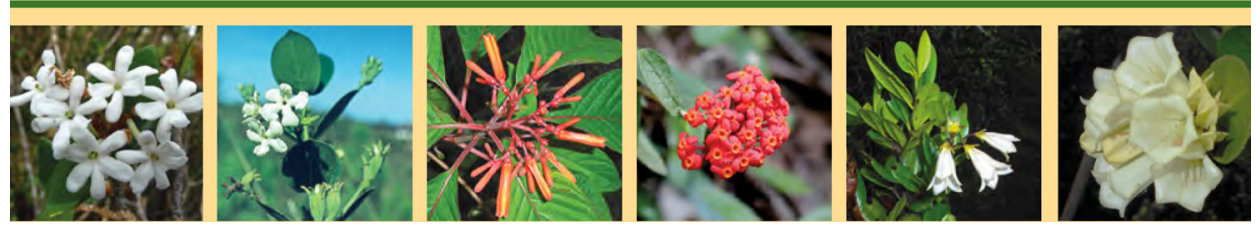

